\title{
The Actual Intrinsic Excitability of Granular Cells Determines the Ruling Neurovascular Coupling Mechanism in the Rat Dentate Gyrus
}

\author{
Frank Angenstein \\ Functional Imaging Group, Deutsches Zentrum für Neurodegenerative Erkrankungen, and Special Laboratory for Noninvasive Brain Imaging, Leibniz \\ Institute for Neurobiology, 39118 Magdeburg, Germany
}

Paired-pulse stimulation of the perforant pathway was used to study the relation between granular cell activity and the resultant fMRI response in the rat dentate gyrus. By varying the interpulse interval (IPI), paired-pulse stimulations caused: a depression (20 ms IPI), a facilitation (100 ms IPI), a mixture of depression and facilitation (30 ms IPI), or no change (500 ms IPS) in the second response. Eight identical paired pulses were applied during one stimulation train and the evoked field potentials and generated fMRI responses were measured simultaneously. Application of consecutive stimulation trains caused time-dependent variations in electrophysiological and fMRI responses, which were characteristic for each stimulus protocol. Depending on the IPI, the magnitude of the fMRI response either correlated strongly with or was apparently unrelated to the spiking or postsynaptic activity of the granular cells. A strong relation between spiking activity and resultant fMRI response was only found when the stimulation protocol caused an increase in the recorded population spike latency. If the latency was decreased, the fMRI response was more closely related to the applied input activity. Perforant pathway fibers monosynaptically activate granular cells, so variations in population spike latencies reflect changes in their intrinsic excitability. Therefore, during increased intrinsic excitability, signaling cascades upstream of the granular cells determine the fMRI response, whereas granular cell activity-related mechanisms control the fMRI response during decreased intrinsic excitability.

Key words: BOLD response; electrophysiology; fMRI; hippocampus; neurovascular coupling

\section{Introduction}

fMRI is a widely used technique to search for brain regions involved in various forms of cognitive processes. The technique visualizes with a high spatial resolution changes in hemodynamic parameters such as blood flow, blood volume, or blood oxygenation [blood oxygenation level-dependent (BOLD)], which in turn are controlled by variations in ongoing neuronal activities in these regions. However, as long as the underlying neurovascular coupling processes are elusive, an unambiguous interpretation of fMRI results that goes beyond the general conclusion of a locally modified neuronal activity remains difficult.

A detailed study of neurovascular-coupling processes requires simultaneous measurements of neuronal activities and the resultant hemodynamic parameters in the same region at the same time. In addition, to modify the ongoing neuronal activity in this region, a defined electrical stimulation of a fiber bundle projecting monosynaptically to this region is a prerequisite. Such an

Received Feb. 3, 2014; revised May 9, 2014; accepted May 9, 2014.

Author contributions: F.A. designed research; F.A. performed research; F.A. analyzed data; F.A. wrote the paper. This work was supported by the Deutsche Forschungsgemeinschaft (Grant An200 - 06). I thank K. Krautwald for excellent technical assistance.

The author declares no competing financial interests.

Correspondence should be addressed to Frank Angenstein, MD, PhD, Deutsches Zentrum für Neurodegenerative Erkrankungen (DZNE), Leipzigerstr. 44, 39118 Magdeburg, Germany. E-mail: frank.angenstein@dzne.de.

DOI:10.1523/JNEUROSCI.0472-14.2014

Copyright $\odot 2014$ the authors $\quad 0270-6474 / 14 / 348529-17 \$ 15.00 / 0$ experimental approach that simultaneously measures fMRI and neurophysiological responses in the dentate gyrus during electrical stimulation of the perforant pathway was developed previously (Angenstein et al., 2007) and proved that the measured fMRI response in the dentate gyrus depends on qualitative changes in local signal processing rather than on the pure input or output activity (Canals et al., 2008; Angenstein et al., 2009). Signal processing comprises several interactions of the principal cells with local acting interneurons (Buhl and Whittington, 2007), as well as changes in the intrinsic activity of all activated neurons. In general, if the input is unchanged and the output changes, it must mean that the incoming stimulus is processed differently. Variations in the processing of incoming signals occur during pairedpulse stimulations. The neuronal response to two succeeding identical pulses critically depends on the interpulse interval (IPI). There is an initial paired-pulse depression, in which the response to the second pulse is smaller compared with the first pulse and has short IPIs (10-30 ms); a paired-pulse facilitation, in which the response to the second pulse is stronger than the first pulse and has intermediate IPIs (40-300 ms); and another paired-pulse depression with long IPIs (>500-1000 ms). Paired-pulse depression induced by short IPIs reflects the $\mathrm{GABA}_{\mathrm{A}}$-mediated feedback inhibition of granular cells through local interneurons (Tuff et al., 1983; Oliver and Miller, 1985; Sloviter, 1991; Lømo, 2009), whereas paired-pulse facilitation is thought to be mediated by an increase of NMDA-mediated responses to further glutamate re- 
lease (McNaughton, 1982; Albertson and Joy, 1987). Pairedpulse depression induced by long IPIs is caused by the development of a slow hyperpolarizing synaptic potential and reduced transmitter release via the activation of presynaptic $\mathrm{GABA}_{\mathrm{B}}$ receptors (Peet and McLennan, 1986). Therefore, by simply varying the IPI, the processing of the second pulse differs substantially. To determine whether the measured BOLD response allows inferences on the underlying neuronal processes, we compared BOLD responses generated during stimulations with paired pulses that caused: (1) depression (20 ms IPIs), (2) facilitation (100 ms IPIs), (3) a mixture of depression and facilitation (30 ms IPI), or (4) no changes (500 ms IPS) in the second response.

\section{Materials and Methods}

Animals and surgical procedure. For electrode implantation, 9- to 10week-old male Wistar rats were anesthetized with pentobarbital (40 mg/ $\mathrm{kg}$, i.p.) and placed into a stereotactic frame. A bipolar stimulation electrode ( $114 \mu \mathrm{m}$ in diameter, made from Teflon-coated tungsten wire) was placed into the perforant pathway in the right hemisphere at the coordinates AP: -6.9 , ML: $4.1 \mathrm{~mm}$ from bregma, DV: $2.0-2.5 \mathrm{~mm}$ from the dural surface. A monopolar recording electrode $(114 \mu \mathrm{m}$ in diameter, made from Teflon-coated tungsten wire) was lowered into the granular cell layer of the right dentate gyrus AP: $-2.8 \mathrm{~mm}$, ML: $1.8 \mathrm{~mm}$ from bregma, DV: $2.8-3.2 \mathrm{~mm}$ from the dural surface. Monitoring the monosynaptic evoked field potentials during implantation controlled the correct placement, especially with regard to electrode depth. Grounding and indifferent electrodes (silver wires) were set on the dura through the left side of the cranium and fixed to the skull with dental cement and plastic screws. After surgery, the animals were housed individually and given $7 \mathrm{~d}$ for recovery with ad libitum food and water. The experiments were approved by the animal care committee of the State Saxony-Anhalt (203.h42502-2-852 IfN).

Combined fMRI and electrophysiological measurements. Detailed descriptions of the experimental setup used for simultaneous fMRI and electrophysiological measurements during electrical stimulation of the perforant pathway has been described previously (Angenstein et al., 2007). For the fMRI experiment, the anesthetized animals were connected to the stimulation and recording electrode after fixation of the head. Heating was provided from the ventral side and heart rate, breathing rate, and oxygen saturation was monitored during the entire experiment using an MRIcompatible pulse oxymeter (MouseOx; Starr Life Sciences).

To determine the appropriate stimulation intensity for the subsequent fMRI experiment, an input/output curve for the population spike was first constructed in each individual animal. For that, the perforant pathway was stimulated with single test pulses at increasing intensities (i.e., 3 test pulses at $10 \mathrm{~s}$ intervals with the following intensities: 100, 200, 300, $400,500,600,800 \mu \mathrm{A}$; recordings were made with an interval of $2 \mathrm{~min}$, except the $600-800 \mu \mathrm{A}$, which were taken at $4 \mathrm{~min}$ intervals). The electrophysiological responses were filtered with an anti-aliasing filter (i.e., a low cutoff filter at $<1 \mathrm{~Hz}$ and a high cutoff filter at $>5000 \mathrm{~Hz}$ ) using an amplifier (EX4-400; Science Products), transformed by an analog-todigital interface (power-CED; Cambridge Electronic Design), and stored on a personal computer with a sampling rate of $13,000 \mathrm{~Hz}$.

The amplitude of the population spike was measured in millivolts (from the first most positive point to the following most negative point; see Fig. 1) and the latency in milliseconds (from the middle of the stimulus artifact to the most negative point). The intensity that evoked $50 \%$ of the maximum population spike amplitude was then used for the subsequent experimental stimulations. Postsynaptic activity was measured as slope of the fEPSP (i.e., the slope through all points obtained on the first positive deflection of the potential).

All fMRI experiments were performed on a $4.7 \mathrm{~T}$ Bruker Biospec 47/20 scanner equipped with a BGA09 $(400 \mathrm{mT} / \mathrm{m})$ gradient system. A $50 \mathrm{~mm}$ Litzcage small animal imaging system (Doty Scientific) was used for radio frequency $(\mathrm{RF})$ excitation and signal reception. Initially, 10 horizontal anatomical spin-echo images $\left(T_{2}\right.$-weighted $)$ were obtained using a rapid acquisition relaxation enhanced (RARE) sequence (Hennig et al., 1986) with the following parameters: TR $4000 \mathrm{~ms}$; TE $15 \mathrm{~ms}$, slice thickness $0.8 \mathrm{~mm}$, FOV $36.5 \times 36.5 \mathrm{~mm}$, matrix $256 \times 256$, RARE factor 8 , averages 4 . The total scanning time was $9 \mathrm{~min}$. $\mathrm{PMRI}$ was performed using an echoplanar imaging sequence with the following parameters: TR 2000 $\mathrm{ms}$, TE $24 \mathrm{~ms}$, slice thickness $0.8 \mathrm{~mm}$, FOV $36.5 \times 36.5 \mathrm{~mm}$, matrix $92 \times$ 92. The slice geometry (i.e., 10 horizontal slices) was identical to the previously obtained anatomical spin-echo images.

Two basic stimulation protocols were used for the fMRI measurement. First, the perforant pathway was electrically stimulated in four consecutive stimulation blocks (Fig. 1A); each block consisted of seven identical stimulation trains. After a 2 min baseline (first block) or 2 min after the last train of the previous stimulation block, the stimulation trains were applied, if not otherwise mentioned, every minute. The number of pulses per second was set to 2 , the IPIs were varied at $20 \mathrm{~ms}$ (consistent pairedpulse inhibition), $30 \mathrm{~ms}$ (mixed paired-pulse facilitation and inhibition), $100 \mathrm{~ms}$ (consistent paired-pulse facilitation), or $500 \mathrm{~ms}$ (or continuous 2 $\mathrm{Hz}$ stimulation). The interval between the first pulses was always $1 \mathrm{~s}$ (Fig. $1 B)$. One stimulation train lasted $8 \mathrm{~s}$ and therefore contained 16 pulses, and was followed by a resting period of $52 \mathrm{~s}$. In one group, the perforant pathway was stimulated with only one pulse per second (continuous $1 \mathrm{~Hz}$ stimulation). Second, the perforant pathway was stimulated with 28 consecutive stimulation trains. Within a train, the stimulation condition remained constant; however, the stimulation protocol changed after each train. During the first train, 8 paired pulses with an IPI of $20 \mathrm{~ms}$ were applied; during the second train, 8 single pulses $(1 \mathrm{~Hz})$; during the third train, 8 paired pulses with an IPI of $100 \mathrm{~ms}$; and during the fourth train, 8 paired pulses with an IPI of $500 \mathrm{~ms}(2 \mathrm{~Hz})$ were applied. This alternating stimulation protocol was repeated seven times (Fig. 1B).

The electrophysiological responses during the fMRI session were filtered with a low cutoff filter $(<1 \mathrm{~Hz})$ and a high cutoff filter $(>5000 \mathrm{~Hz})$ using an amplifier (EX4-400; Science Products), transformed by an analog-to-digital interface (power-CED; Cambridge Electronic Design), and stored on a personal computer with a sampling rate of $5000 \mathrm{~Hz}$. The lower sampling rate did not affect the shape of the recorded population spike (i.e., compared with the initial response measured during the input-output curve) because the underlying signals did not contain frequencies $>1 \mathrm{kHz}$.

Data analysis. The functional data were loaded and converted into a BrainVoyager data format. A standard sequence of preprocessing steps implemented in the BrainVoyager QX software (Brain Innovation) such as 3D motion correction (trilinear interpolation and reduced data using the first volume as a reference) and temporal filtering (high pass GLMFourier: 3 sines/cosines and Gaussian filter, FWHM 3 data points) was applied to each dataset. No slice time correction algorithm was applied. Significant activation was only found in two of 10 slices, so analysis with and without slice time correction resulted in a similar activation pattern. Because the reconstruction of the $\mathrm{fMR}$ images resulted in a $128 \times 128$ matrix (instead of the $92 \times 92$ imaging matrix), a spatial smoothing (Gaussian filter of 1.4 voxel) was added. Functional activation in each individual animal was analyzed using the correlation of the observed BOLD signal intensity changes in each voxel with a predictor (hemodynamic response function) generated from the given stimulus protocol (see Combined fMRI and electrophysiological measurements, above). Based on this, the appropriate activation map could be generated (Fig. 1A). To calculate the predictor, the square wave representing stimulus on and off conditions was convolved using a double gamma hemodynamic response function (onset $0 \mathrm{~s}$, time to response peak $5 \mathrm{~s}$, time to undershoot peak $15 \mathrm{~s}$ ). To exclude false-positive voxels, we only considered those with a significance level of $p<6.8 \times 10^{-7}\left(t_{\min }=5\right)$ for analysis of the size of the activated area, thus clearly above the threshold set by calculating the false discovery rate (FDR) with a $q$-value of 0.05 (which corresponds to a $t$ value $<3$ or $p<$ $0.005)$. The BOLD time series depicted for each region represent variations in the BOLD signal intensities of all significantly activated voxels in the corresponding region of interest. The BOLD time series shown in all figures are the averages of all of the BOLD signal time series measured in all of the individual animals \pm SD.

Event-related BOLD responses were calculated by measuring the signal intensities starting 6 frames before stimulus onset ( $-12 \mathrm{~s}$ until $0 \mathrm{~s})$, 


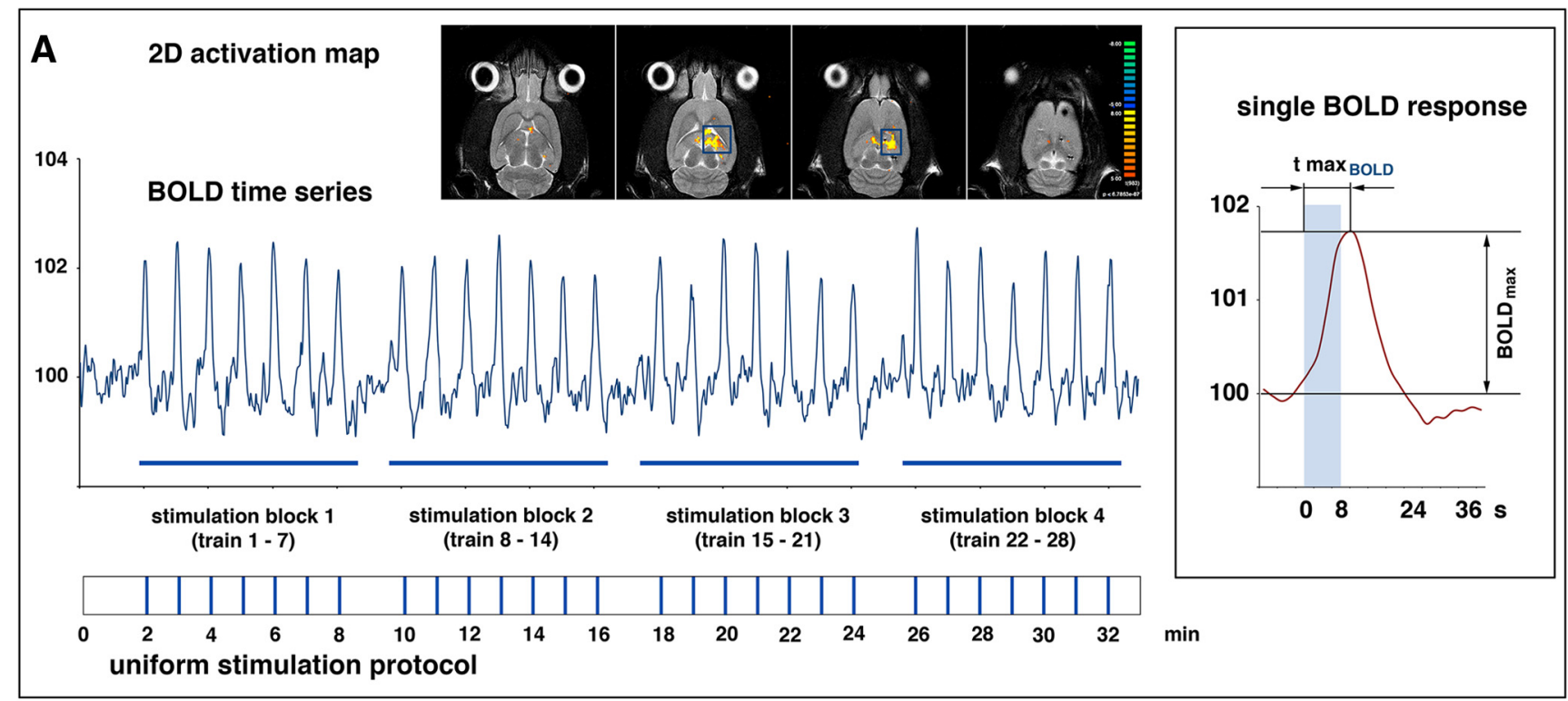

\section{B alternate stimulation protocol}
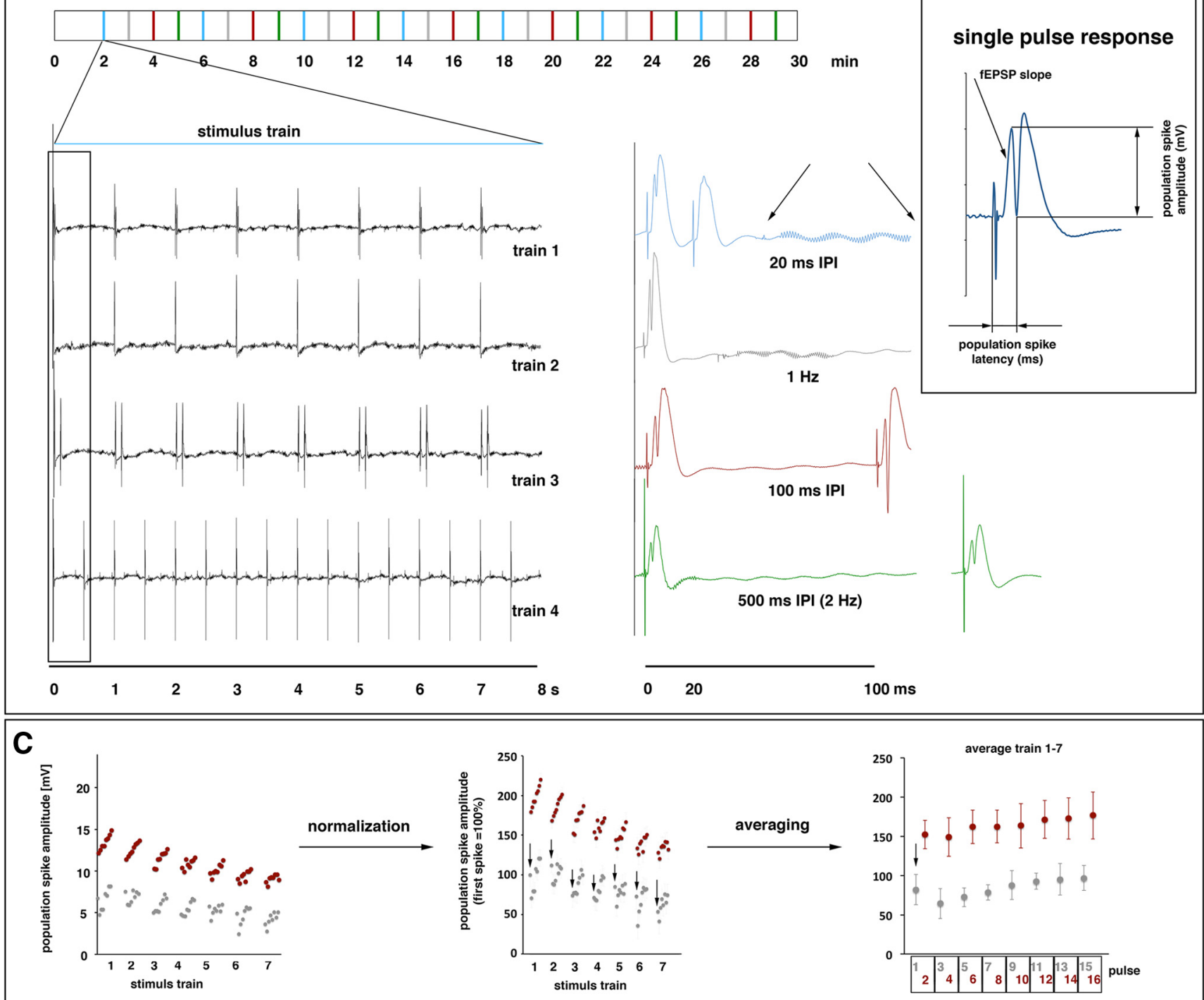

Figure 1. Comparison of simultaneous recorded fMRI data and field potentials in the rat dentate gyrus during electrical stimulations of the right perforant pathway. $\boldsymbol{A}$, Uniform stimulation protocol. The perforant pathway was stimulated with four consecutive identical stimulation blocks. Each stimulation block consisted of seven stimulation trains. During the entire stimulation session, BOLD signal intensities were measured and the BOLD time series of all significantly activated voxels in the right dentate gyrus region (blue boxes) were calculated. The maximal value (Figure legend continues.) 
during stimulus presentation (between 0 and $8 \mathrm{~s}$, which corresponds to 4 frames), and the following 20 frames ( 8 to $48 \mathrm{~s}$ ) after the end of the stimulus (Fig. 1A). To avoid the confounding effect of putative variations in baseline BOLD signal intensities on the calculated BOLD response (i.e., BOLD signal stimulus $_{\text {BOLD signal }}$ baseline ${ }^{\star} 100 \%$ ), each BOLD response was related to BOLD signal intensities of the stimulus over the preceding $12 \mathrm{~s}$. To compare average maximal BOLD responses between conditions, a two-tailed unpaired Student's $t$ test was performed. To compare the average maximal BOLD responses during consecutive stimulation blocks or between different stimulation conditions during the alternate stimulation protocol, a two-tailed Wilcoxon signed-rank test was performed. Differences were considered significant at a calculated $p$-value $<0.05$.

To summarize the spatial distribution of significantly activated voxels during a particular stimulation condition, all fMRI datasets were aligned to a 3D standard rat brain using anatomical landmarks. These datasets were then further analyzed with a linear regression analysis using a general linear model (GLM) and multisubject analysis implemented in BrainVoyager QX software. To provide a conservative representation of the main effects, the significance level was set to $t_{\min }=6\left(p<7.2 \times 10^{-9}\right)$. All significantly activated voxels were converted into volumes of interest (VOI), from which surface clusters were created and visualized by rendering using the BrainVoyager "VOI analysis tool."

The correlation between electrophysiological measured parameters (population spike amplitude, population spike latency, field EPSP slope) and maximal BOLD response was calculated with a free statistics software package from the Office for Research Development and Education (http://www.wessa.net/rwasp_correlation.wasp).

Electrophysiological data were normalized to the response to the first pulse $(100 \%)$ and plotted as relative changes (Fig. 1C). To visualize the development of the responses during consecutive stimulation blocks, as well as the development of the responses within a train (see Figs. 2, 3, 4, 5), the corresponding responses in all trains of one stimulation block were averaged [i.e., average first response $=$ (pulse $1_{\text {train } 1}+$ pulse $1_{\text {train2 }}+$ pulse $1_{\text {train } 3}+\ldots+$ pulse $\left.1_{\text {train } 7}\right) / 7$ ); Fig. $\left.1 C\right]$.

\section{Results}

Repetitive electrical stimulation of the perforant pathway often results in long-lasting modifications of local neuronal network properties, resulting in elicited neuronal responses to later stimulation trains that may differ from the initial stimulation trains and, as a result, the resultant BOLD response (Angenstein et al., 2009; Angenstein et al., 2010). Therefore, we performed three sets of experiments. In the first set of experiments, only one stimulus

\footnotetext{
$\leftarrow$

(Figure legend continued.) of $\mathrm{BOLD}$ signal intensities $\left(\mathrm{BOLD}_{\max }\right)$ and the time to reach this value ( $t$-max $\left.{ }_{B O L D}\right)$ were used to describe the individual BOLD response to each individual stimulus train (right inset). $\boldsymbol{B}$, Alternate stimulation protocol. The perforant pathway was stimulated with 28 consecutive stimulation trains; however, the stimulation protocol changed after each train. During the first train, eight paired pulses with an IPI of $20 \mathrm{~ms}$ were applied. This stimulation condition caused a consistent paired-pulse inhibition (field recordings of the first $100 \mathrm{~ms}$ of the train is depicted on the right). During the second train, eight single pulses were applied. This stimulation condition elicited eight similar electrophysiological responses. During the third train, eight paired pulses with an IPI of $100 \mathrm{~ms}$ were applied. This stimulation condition caused consistent paired-pulse facilitations. During the fourth train, 16 pulses with a constant IPI of $500 \mathrm{~ms}$ (i.e., a continuous $2 \mathrm{~Hz}$ stimulation) were applied. This stimulation condition did not induce consistent variations in the responses between two consecutive pulses. This circle of four stimulation conditions was repeated seven times. To describe the electrophysiological response to each individual pulse, the population spike amplitude, population spike latency, and the initial slope (fEPSP slope) were determined (right inset). $\boldsymbol{C}$, Visualization of the electrophysiological responses. For clarity, the responses to the first pulse per second are marked in gray and the second pulses applied after a specific IPI are marked in a particular color. The calculated raw data (e.g., population spike amplitudes in millivolts, left) were normalized to the first measured response, which is set to $100 \%$ (middle). Then, the corresponding responses in each train (e.g., all first responses indicated by small black arrows) were averaged and summarized as average response per stimulation block (right).
}

condition was applied and the resultant BOLD responses in each animal of one group were averaged and compared with the averaged BOLD response observed in another group of animals stimulated with a different stimulus protocol. In the second and third sets of experiments, different stimulus protocols were applied to the same animal in one session and then the resultant BOLD responses were compared (Fig. 1).

\section{Electrophysiological and resultant BOLD responses in the dentate gyrus during repetitive stimulations of the perforant pathway with 1 and $2 \mathrm{~Hz}$ stimulation trains}

Previous work (Krautwald and Angenstein, 2012) has shown that, under medetomidine, stimulation of the perforant pathway with 1 pulse per second $(1 \mathrm{~Hz})$ or higher generates significant BOLD responses in the dentate gyrus. To relate the paired-pulse stimulations to the general stimulus pattern (one paired pulse per second) and to the number of pulses (2 pulses per second), we first measured the electrophysiological and BOLD responses to a continuous 1 and $2 \mathrm{~Hz}$ stimulation protocol. In agreement with the previous work, stimulation of the perforant pathway with 8 pulses per train $(1 \mathrm{~Hz})$ resulted in the generation of significant BOLD responses in the dentate gyrus (Fig. $2 A$ ). The individual $B O L D$ responses gradually declined during repetitive stimulations; therefore, the BOLD responses observed during the third and fourth stimulation block were significantly smaller than the BOLD responses observed during the first stimulation block (Fig. $2 A$, Table 1). Similarly, the average population spike amplitude per train significantly decreased from the first to the fourth stimulation block (Fig. 2B, Table 1). As a result of the similar developments of the population spike amplitude and maximal BOLD response, a moderate correlation was found between these two different parameters of neuronal activity $\left(R^{2}=0.550, t_{(26)}=\right.$ $4.232, p<0.0001$; Fig. $2 C$ ). In contrast to the population spike amplitude, the average population spike latency remained almost constant during repetitive stimulations; therefore, no significant correlation was found between population spike latency and BOLD response $\left(R^{2}=0.210, t_{(26)}=2.625\right.$; Fig. $\left.2 B\right)$. In addition to the parameters of the granular cell spiking, the slope of the fEPSP was determined to relate the postsynaptic activity of the granular cells with the measured BOLD response. The average slope of the fEPSP measured during the first stimulation block (trains 1-7) was similar to the average slope of the fEPSP measured during the last stimulation block (trains 22-28); therefore, the significant decrease of the BOLD responses during last block was not accompanied by changes in the slope of the fEPSP $\left(R^{2}=\right.$ $0.008, t_{(26)}=0.464$; Fig. $2 B$, Table 1$)$.

In addition to the slowly developing changes in the population spike amplitude, which developed independently of the latency, rapidly developing changes in electrophysiological responses were observed during each individual train. In general, the latency significantly increased from the first to the second pulse and then remained on an almost similar elevated level (107.8 \pm $0.4 \%$ ) during the following 7 pulses within the train. This change was not accompanied by a concurrent change in the population spike amplitude or fEPSP slope (Fig. 2B).

In summary, repetitive trains with eight pulses per train affected the signal processing in the dentate gyrus in two different ways: first, by an increase in the population spike latency during each individual stimulation train and, second, by a decrease of the population spike amplitude during consecutive stimulation trains. In contrast to the spiking activity, the postsynaptic activity (i.e., the slope of the fEPSP) remained unchanged during all consecutive stimulation trains. Therefore, from all of the measured 

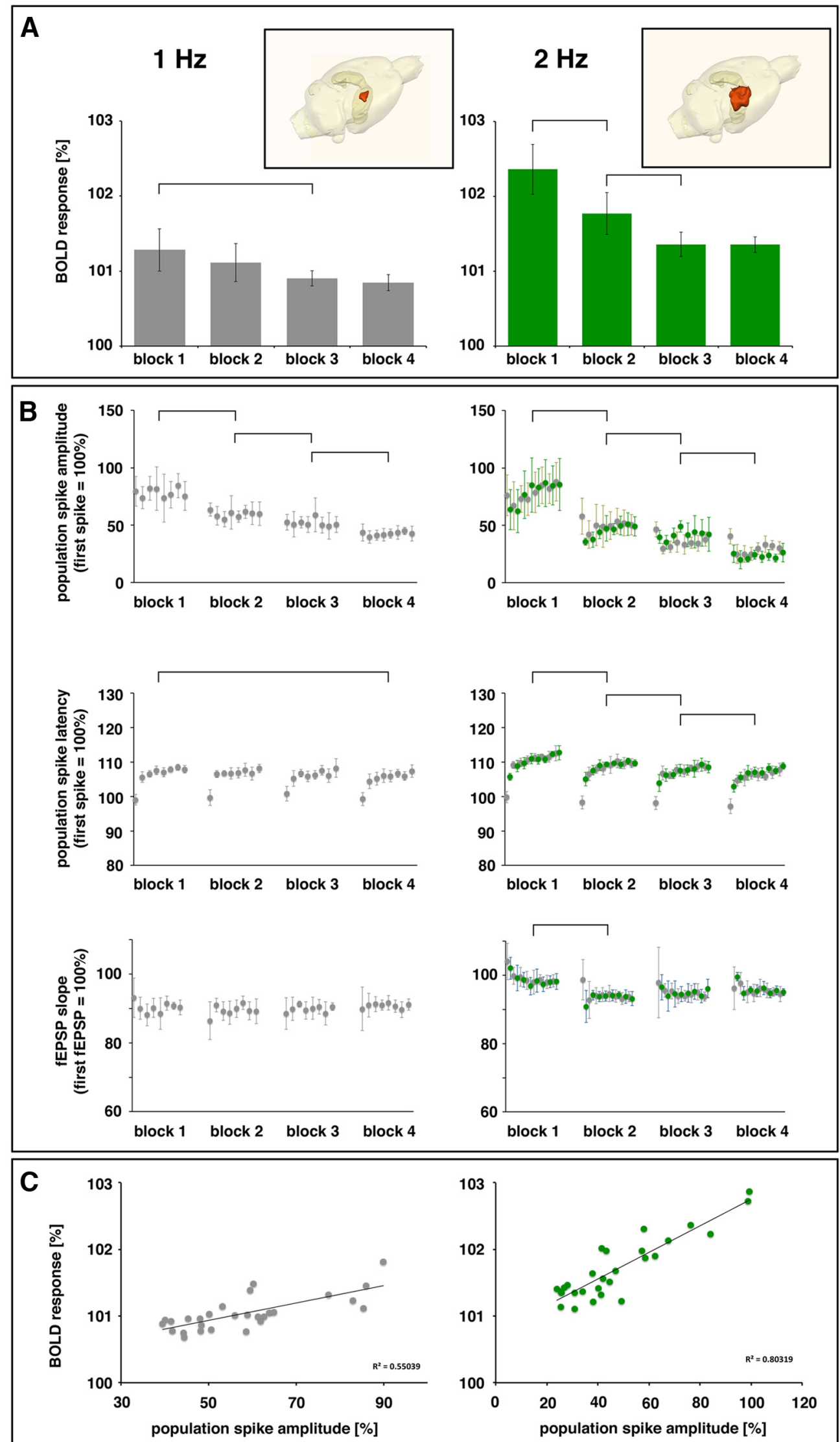

Figure 2. Electrical stimulation of the right perforant pathway with 28 consecutive $1 \mathrm{~Hz}(n=6)$ or $2 \mathrm{~Hz}(n=5)$ stimulation trains as depicted in Figure 1. A, The two stimulation protocols generated significant BOLD responses in the right dentate gyrus, but with different distributions. To visualize the effect of repetitive stimulations, the average BOLD responses of each seven consecutive stimulation-trains (i.e., blocks 1-4) were calculated. During repetitive stimulations with identical $1 \mathrm{~Hz}$ stimulation trains, the generated BOLD response slowly (Figure legend continues.) 
Table 1. Electrophysiologically measured parameters of granular cell activity and corresponding maximal BOLD responses during repetitive electrical stimulations of the perforant pathway

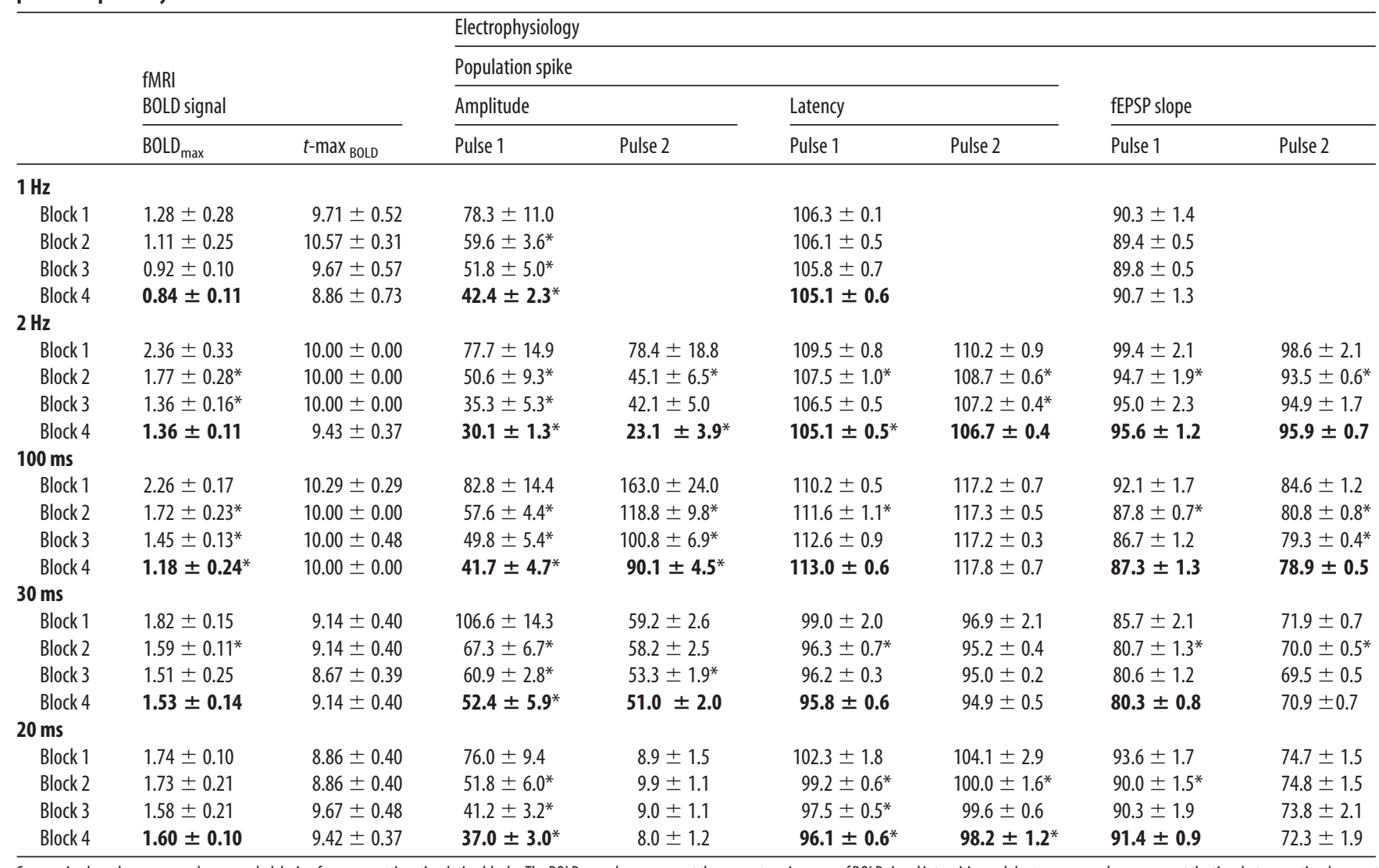

Summarized are the average values recorded during four consecutive stimulation blocks. The BOLD ${ }_{\max }$ values represent the percentage increase of $B O L D$ signal intensities and the $t-$ max $_{B O L D}$ values represent the time between stimulus onset and maximal BOLD response (in seconds). All electrophysiological data are relative values and related to the first response (=100\%).

Asterisks indicate a significant change $(p<0.05)$ to the preceding block and bold numbers indicate significant changes between the first and last stimulation block.

parameters of granular cell activity, only the population spike amplitude appeared to be related to the magnitude of the BOLD response.

Stimulation of the perforant pathway with 16 pulses per train ( $2 \mathrm{~Hz}$ ) induced BOLD responses in the dentate gyrus that were significantly stronger than those during stimulation with 8 pulses per train (Fig. 2A, Table 1). Similar to the $1 \mathrm{~Hz}$ stimulation protocol, individual BOLD responses declined with consecutive stimulations, but with a higher rate (Fig. 2A). Concordantly, the

(Figure legend continued.) decreased; therefore, during the third stimulation block, the average $B O L D$ response was significantly smaller than during the first stimulation block. In contrast, repetitive stimulations with identical $2 \mathrm{~Hz}$ stimulation trains resulted in an initially stronger $B O L D$ response that also declined more rapidly during consecutive blocks. $\boldsymbol{B}$, Concurrent measured field potentials in the dentate gyrus revealed a significant reduction of the population spike amplitude during all consecutive stimulation trains during the two stimulation conditions. Gray dots indicate the first responses per second and the green dots indicate the responses to the second pulse applied with an IPI of $500 \mathrm{~ms}$. Within all individual stimulation trains, the population spike latency significantly increased after the first pulse. The latency of the first population spike did not vary during the entire stimulation protocol. Only during the $2 \mathrm{~Hz}$ stimulation protocol did the average population spike latency decrease significantly during consecutive trains. In contrast to the population spike amplitude, the fEPSP slopes remained almost unchanged during the stimulations. There was only an initial decline from the first to the second stimulation block during the $2 \mathrm{~Hz}$ stimulation protocol. C, Correlation of simultaneously measured BOLD responses and spiking of the granular cells. For each of the 28 stimulation trains, the maximal BOLD response and the corresponding averaged population spike are plotted (first response $=100 \%$ ). During repetitive $1 \mathrm{~Hz}$ stimulation trains, only a moderate correlation was found was found between population spike amplitude and maximal BOLD response; during $2 \mathrm{~Hz}$ stimulations, a strong correlation was found. average population spike amplitude per train decreased significantly from the first to the fourth stimulation block (Fig. $2 B$, Table 1). Because the average population spike amplitude and the magnitude of the corresponding BOLD response showed a very similar development during consecutive stimulations, the correlation between the two parameters was very close $\left(R^{2}=0.803\right.$, $t_{(26)}=10.301, p<0.0001$; Fig. $2 C$ ). In contrast to the $1 \mathrm{~Hz}$ stimulation protocol, the average population spike latency also decreased during the $2 \mathrm{~Hz}$ stimulation protocol; therefore, a strong correlation was also found between these two parameters $\left(R^{2}=0.726, t_{(26)}=8.300, p<0.0001\right.$; Fig. $\left.2 B\right)$. The corresponding fEPSP slopes only decreased after the first stimulation block and then remained almost stable; therefore, variations in the maximal BOLD response were again not paralleled with significant changes in postsynaptic responses $\left(R^{2}=0.340, t_{(26)}=\right.$ 3.659; Fig. 2B).

In addition to the slowly developing changes in the signal processing, fast changes also occurred during each individual train. First, the population spike amplitude dropped from the first to the second pulse and then recovered to reach the initial value. Concurrently, the population spike latency strongly increased from the first to the second pulse and increased subsequently with a lower rate to a final value of $109.8 \pm 1.8 \%$ (Fig. $2 B)$. Although the population spike latency increased significantly during each train, the latencies of first population spikes in each train remained similar during all consecutive trains (Fig. $2 B)$. The changes in the population spikes in one train were accompanied by minor changes in the fEPSP slope. 
In summary, the $2 \mathrm{~Hz}$ stimulation protocol affected the signal processing in the dentate gyrus in the course of the entire experiment in a more complex manner than the $1 \mathrm{~Hz}$ stimulation protocol. First, short-lasting changes in the signal processing were induced, as reflected by specific changes of the population spike amplitude and latency within one train. Second, longerlasting changes in signal processing (i.e., differences in the population spikes observed between consecutive trains) resulted in a significant decrease in the population spike amplitude and latency. Whereas the long-lasting changes in the population spike amplitude and latency were concordant, short-lasting changes in the development of the two parameters were not in agreement.

Based on these results, the stronger BOLD responses during 2 $\mathrm{Hz}$ stimulation compared with $1 \mathrm{~Hz}$ stimulations could be explained by the presence of: (1) a double input (synaptic) activity, (2) a double output (granular cell spiking) activity, (3) an increased complexity of the signal processing, or (4) any combination of these 3 factors.

\section{Electrophysiological and resultant BOLD responses in the dentate gyrus during repetitive stimulations of the perforant pathway with identical paired pulses}

To discern between the roles of local signal processing and synaptic and spiking activity for the generation of the BOLD response, the same stimulation protocol was repeated but with 3 different paired-pulse stimulus conditions; therefore, the numerical input activity (16 pulses) was kept identical to the $2 \mathrm{~Hz}$ stimulation protocol and the pattern (1 paired-pulse per second) identical to the $1 \mathrm{~Hz}$ stimulation protocol.

Repetitive stimulations with paired pulses having an IPI of 20 $\mathrm{ms}$ also generated significant BOLD responses in the right DG (Fig. 3A). The magnitude of the BOLD responses to individual stimulation trains only decreased slightly during the course of the experiment (Fig. 3A, Table 1).

The stimulation protocol resulted in a clear and constant paired-pulse inhibition; that is, the second population spike amplitude was always either smaller or even absent (Fig. 3B). In general, the average population spike amplitude and latency decreased during consecutive stimulation trains; therefore, they were significantly smaller during the last stimulation block than at the beginning. In contrast, the slope of the fEPSP only decreased after the first stimulation block and then remained at an almost constant level (Fig. 3B). Because the BOLD response remained almost unchanged while the population spike amplitude and latency decreased significantly during consecutive stimulation trains, there was no significant relation between the population spike amplitude $\left(R^{2}=0.157, t_{(26)}=2.201\right)$ or population spike latency $\left(R^{2}=0.078, t_{(26)}=1.483\right)$ and the resultant maximal BOLD response under this stimulus condition (Fig. $3 C$ ). Similarly, the development of the BOLD response did not coincide with variations in the slope of the fEPSPs (Fig. $3 B$ ); therefore, no correlation was found between the slope of the fEPSPs and the corresponding BOLD responses $\left(R^{2}=0.010, t_{(26)}=0.512\right)$.

In addition to these general changes in neuronal responses to consecutive stimulation trains, the repeated application of eight consecutive paired pulses during one train resulted in a complex response pattern. Considering only the population spikes induced by the first pulses of each pair, the development of the population spike amplitudes matched the development of the corresponding pulses during the $2 \mathrm{~Hz}$ stimulation protocol (Figs. $2 B, 3 B)$. In contrast, the average population spike latencies were significantly smaller (Table 1). Although the population spike latencies also increased within the first stimulation train, the av- erage population spike latency decreased during the subsequent trains. At the end of the first stimulation block, the latencies of the population spike only increased to $101.5 \pm 0.9 \%$ and, during the last block, only to $98.5 \pm 1.2 \%$. In contrast to the $2 \mathrm{~Hz}$ stimulation protocol, the latency of the first population spike in each train was not stable, but dropped to $91.0 \pm 0.9 \%$ during the last stimulation block (Fig. 3B).

The $20 \mathrm{~ms}$ IPI protocol generated BOLD responses that were not related to any of the measured parameters of neuronal activities. In contrast to the 1 or $2 \mathrm{~Hz}$ stimulation protocol, repetitive stimulations with paired pulses with $20 \mathrm{~ms}$ IPI caused a clear reduction of the average population spike latency.

Consecutive paired pulses with an IPI of $100 \mathrm{~ms}$ also generated significant BOLD responses in the dentate gyrus (Fig. 3A). Similar to the $2 \mathrm{~Hz}$ stimulation protocol, the BOLD responses decreased with each consecutive stimulation train such that clearly weaker BOLD responses were generated during the last stimulation block than during the first (Fig. 3A, Table 1).

Repetitive paired-pulse stimulation with an IPI of $100 \mathrm{~ms}$ resulted in a consistent facilitation of the response to the second pulse (Fig. $3 B$ ). The average population spike amplitudes per train significantly decreased during all consecutive stimulation blocks (Fig. 3B, Table 1). The average population spike latency did not decrease during consecutive stimulation trains, but instead increased. In particular, the latency of the population spikes to the first pulse increased, whereas the latencies to the corresponding second pulses remained almost constant (Table 1).

Concurrent with the changes in the population spike amplitude and latency, the fEPSP slope decreased only after the first stimulation block stimulation trains. Therefore, the average fEPSP slope during the last stimulation block was significantly smaller than the average fEPSP slope during the first stimulation block (Fig. 3B). Relating the average population spike amplitude and latency to the resultant maximal BOLD response revealed a very close correlation of the BOLD response with the population spike amplitude $\left(R^{2}=0.819, t_{(26)}=10.855, p<0.0001\right)$ and an indirect correlation with the population spike latency $\left(R^{2}=\right.$ $0.679, t_{(26)}=-7.410, p<0.0001$; Fig. $3 C$ ). In addition, the slope of the fEPSP correlated with the concurrently generated BOLD response $\left(R^{2}=0.713, t_{(26)}=8.034, p<0.0001\right)$. Therefore, during this stimulation condition, the synchronized spiking activity, as well as the postsynaptic activity of the granular cells, both appeared to be good predictors for the magnitude of the generated BOLD response.

Also during this stimulation condition, the induced neuronal responses to the presented eight consecutive paired pulses varied within a train. Considering only the population spikes elicited by the first pulses, the development of the population spike amplitude and latencies was similar to the corresponding pulses during the $2 \mathrm{~Hz}$ stimulation protocol (Figs. $2 B, 3 B$ ). In contrast, significantly increased amplitudes and latencies characterized the responses to the corresponding second pulses. Therefore, compared with the $2 \mathrm{~Hz}$ stimulation protocol, paired-pulse stimulation with IPI of $100 \mathrm{~ms}$ resulted in an overall increased granular cell-spiking activity with increased population spike latencies. Similar to the $2 \mathrm{~Hz}$ stimulation protocol, the latencies of the first pulse in each train were maintained at the initial value (102.9 \pm $0.9 \%)$, whereas at the end of each train, the population spike latencies increased to $114.1 \pm 1.7 \%$ (first pulse) and $118.7 \pm$ $0.2 \%$ (second pulse; Fig. $3 B$ ).

The $100 \mathrm{~ms}$ IPI protocol generated BOLD responses that were related to all of the measured parameters of granular cell activity. 

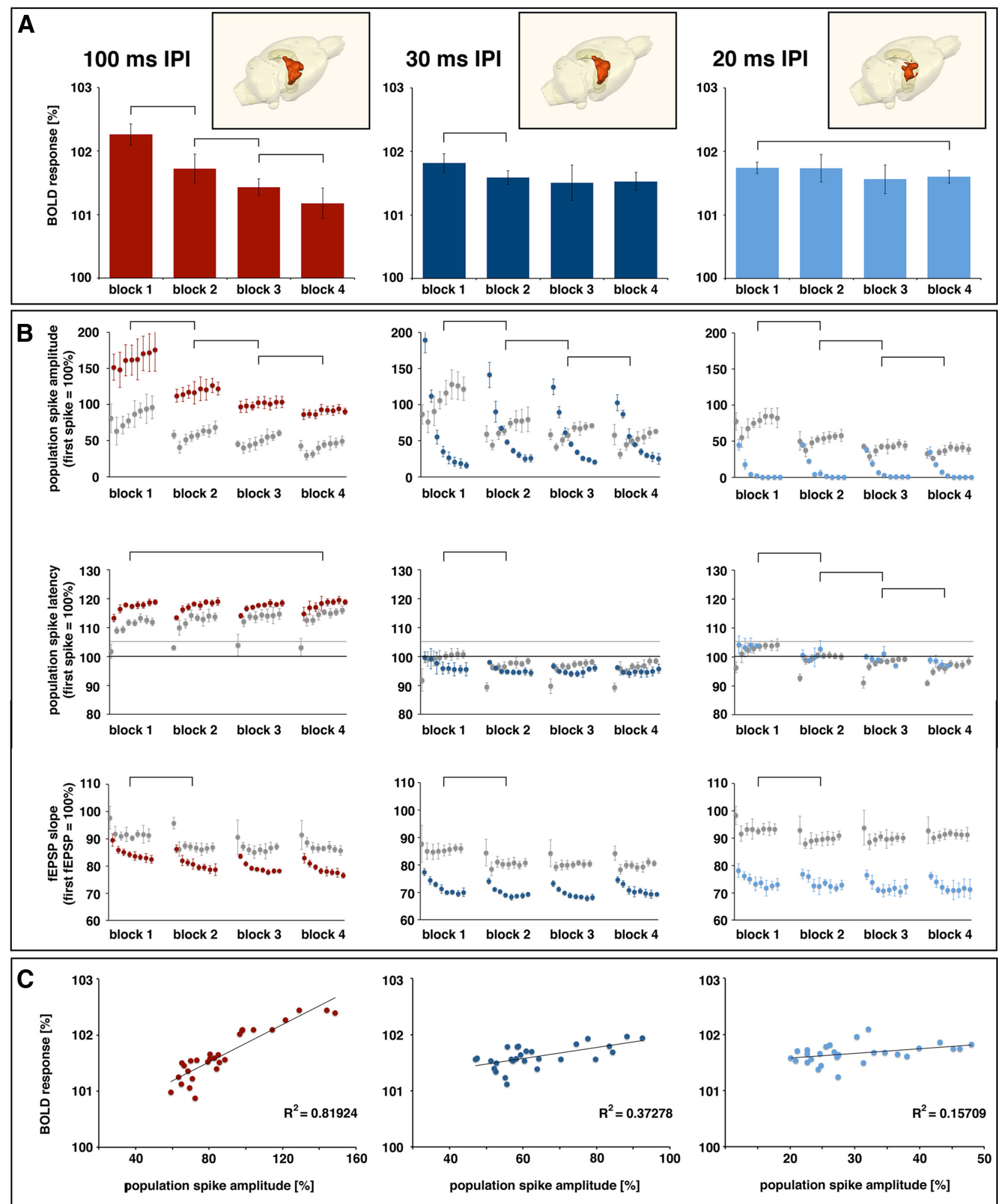

Figure 3. Electrical stimulation of the right perforant pathway with 28 consecutive stimulation trains as depicted in Figure 1. One stimulation train consisted of 8 paired pulses with an IPI of 100 $\mathrm{ms}($ left, $n=5)$, $30 \mathrm{~ms}$ (middle, $n=5$ ), or $20 \mathrm{~ms}$ (right, $n=6$ ). $A$, These stimulation protocols generated significant BOLD responses in the right dentate gyrus region. The average BOLD response significantly declined from that seen during consecutive stimulation blocks, when paired pulses with an IPI of $100 \mathrm{~ms}$ were reported. In contrast, the average BOLD responses induced by paired pulses with shorter IPI were more uniform. $\boldsymbol{B}$, Concurrently measured field potentials in the dentate gyrus again revealed a significant reduction of the average population spike amplitude during all consecutive stimulation trains for all conditions. Gray dots indicate the responses to the first pulse per second and the colored dots the responses to the second pulse per second. Again, during all stimulation conditions, the population spike latencies increased after the first pulse in each train. Repetitive paired pulses stimulations with an IPI of $100 \mathrm{~ms}$ resulted in an increase of the average population spike latency. In contrast, repetitive paired-pulse stimulations with short IPIs caused a reduction of the population spike latency. The development of the fEPSP slopes was similar for all three conditions: a significant initial decline was only reported after the first stimulation block. C, Correlation between population spike amplitude and maximal BOLD response only revealed a strong relation between BOLD response and granular cell activity during paired-pulse stimulations with long IPIs. 
This stimulation protocol induced an increase in the average population spike latency.

Repetitive stimulations of the perforant pathway with paired pulses with an IPI of $30 \mathrm{~ms}$ also generated significant BOLD responses in the dentate gyrus (Fig. 3A). In contrast to the $20 \mathrm{~ms}$ IPI protocol, there was already an initial significant decline in the BOLD responses after the first stimulation block.

This stimulation protocol resulted in a more complex neuronal response pattern. First, within each stimulation train, the first two paired pulses resulted in facilitations, whereas the subsequent paired pulses resulted in inhibitions (Fig. 3B). The average population spike amplitude and latency only decreased from the first to the second block and then remained at an almost constant low level. Similarly, the fEPSPs only declined after the first stimulation block (Fig. 3B, Table 1).

Relating again the measured electrophysiological responses to the concurrent induced BOLD response revealed no correlations with the population spike amplitude $\left(R^{2}=0.373, t_{(26)}=3.931\right.$; Fig. $3 C)$, the population spike latency $\left(R^{2}=0.213, t_{(26)}=2.653\right)$, or the fEPSP $\left(R^{2}=0.311, t_{(26)}=3.432\right)$.

In each train, the eight consecutive paired pulses with an IPI of $30 \mathrm{~ms}$ triggered variable population spike amplitudes and latencies. Considering only the population spikes of each of the first pulses, the development of the population spike amplitude and latencies differed from the corresponding pulses during the $2 \mathrm{~Hz}$ stimulation protocol (Fig. 2B). The general development of the population spike amplitude of all eight first pulses was similar: a reduction after the first pair, followed by an increase. However, in contrast to the $2 \mathrm{~Hz}$ stimulation protocol, this increase was more pronounced such that the response at the end of each stimulation train was significantly stronger than at the beginning. The responses to the second pulses of each pair started with a clear facilitation and ended with a clear inhibition. This pattern remained stable during all four consecutive stimulation trains (Fig. $3 B$ ). The development of the latencies of the two corresponding population spikes in each train differed. The latencies of the first population spikes increased again from the first to the second paired pulse and then remained on an almost similar elevated level. The latencies of the corresponding second responses decreased after the third paired pulse. The general time course of the population spike latencies was similar to the paired-pulse protocol with $20 \mathrm{~ms}$ IPIs. At the end of the first stimulation block, the latencies of the population spike only increased to $95.2 \pm 2.5 \%$ and, during the last block, only to $98.5 \pm 0.8 \%$. Similar to the 20 ms IPI protocol, the latencies of the first population spikes decreased during repetitive stimulation to $89.2 \pm 1.5 \%$ during the last stimulation block. Therefore, also under this stimulation condition, the population spike latencies did not increase above the initial value.

The $30 \mathrm{~ms}$ IPI protocol generated BOLD responses that were not closely related to any of the measured parameters of neuronal activities. This stimulation protocol again caused a decrease in the average population spike latencies during consecutive stimulations.

In general, all of the paired-pulse stimulation protocols used resulted in a time-dependent decrease of population spike amplitude during the entire experiment (i.e., during all consecutive 28 stimulation trains) that resembled the development of the population spike amplitude during the $1 \mathrm{~Hz}$ stimulation protocol. In contrast, the decline of the resultant BOLD responses varied depending on the used stimulation protocol. There was, on one side, a strong decline during repetitive trains of pulses with 100 ms IPI (similar to the $2 \mathrm{~Hz}$ protocol), and on the other side, only a minor decline during repetitive trains with short IPIs (i.e., 30 and $20 \mathrm{~ms}$ ). Therefore, the correlation between granular cellspiking activity and BOLD response was strong during stimulations with long IPIs (i.e., 100 or $500 \mathrm{~ms}$ ), but absent during stimulations with short IPIs (i.e., 20 or $30 \mathrm{~ms}$ ). The presence or absence of a strong correlation between spiking activity and BOLD response coincides with the presence or absence of a significant increase in the population spike latency above the initial value. The average population spike latency only increased during repetitive paired-pulse stimulations with long IPIs, but decreased during repetitive paired-pulse stimulations with short IPIs. The slope of the fEPSP as a measure for postsynaptic activity only correlated with the magnitude of the BOLD response during the $100 \mathrm{~ms}$ IPI protocol; that is, only under a condition in which the slope of the fEPSP strongly correlated with the population spike amplitude.

Because the decline of the BOLD responses during consecutive stimulation trains is stimulus dependent, the comparison of the resultant BOLD responses depends on the number of stimulation trains. When the BOLD response is calculated by averaging the responses to all trains (i.e., trains 1-28), then all stimulation protocols using 16 pulses per train (i.e., $2 \mathrm{~Hz}$, paired pulses with an IPI of 20,30, and $100 \mathrm{~ms}$ ) generated similar BOLD responses, which were all significantly stronger than the BOLD response generated by the $1 \mathrm{~Hz}$ stimulation protocol. Considering only the first stimulation block (i.e., trains 1-7), paired-pulse protocols that caused facilitation generated stronger BOLD responses than paired-pulse protocols that induced an inhibition. In addition to a stronger BOLD response, the time to reach the maximal BOLD signal intensity was significantly longer and corresponded to the values observed during the $1 \mathrm{~Hz}$ stimulation protocol (Fig. $4 \mathrm{~A}$, Table 1). However, at later time points during the last stimulation block (or trains 21-28) when the granular cell spiking activity was adapted to a lower level, paired-pulse stimulation protocols that caused a facilitation generated smaller BOLD responses than paired-pulse stimulation that caused an inhibition (Fig. 4A). At this time point, the time to reach the maximal BOLD signal intensity was similar for all conditions (Table 1).

\section{Electrophysiological and resultant BOLD responses in the dentate gyrus during stimulation of the perforant pathway with two different stimulation protocols}

In a second set of experiments, the perforant pathway was again stimulated with four consecutive stimulation blocks. The stimulation always started with 7 trains of 16 pulses with a constant IPI of $500 \mathrm{~ms}$ (i.e., continuous $2 \mathrm{~Hz}$ stimulation protocol). During the next block, the perforant pathway was stimulated with eight paired pulses per train, the stimulation protocol was switched back to the initial continuous $2 \mathrm{~Hz}$ protocol, and then the same paired-pulse protocol as during the second block was applied during the last block.

Continuous $2 \mathrm{~Hz}$ stimulation trains triggered significant BOLD responses, as observed in the first set of experiments. Again, the average population spike amplitude decreased from train to train and the $2 \mathrm{~Hz}$ stimulation protocol caused an increase in the population spike latencies.

Changing the stimulation protocol during the second block to paired-pulse stimulations with an IPI of $100 \mathrm{~ms}$ resulted in a similar BOLD response. The resulting hemodynamic response function was almost identical (Fig. 5A, Table 2); that is, the time to reach the maximal BOLD signal intensity was similar. The population spike amplitudes of the first responses in each pair further declined with consecutive trains in the second block. The 

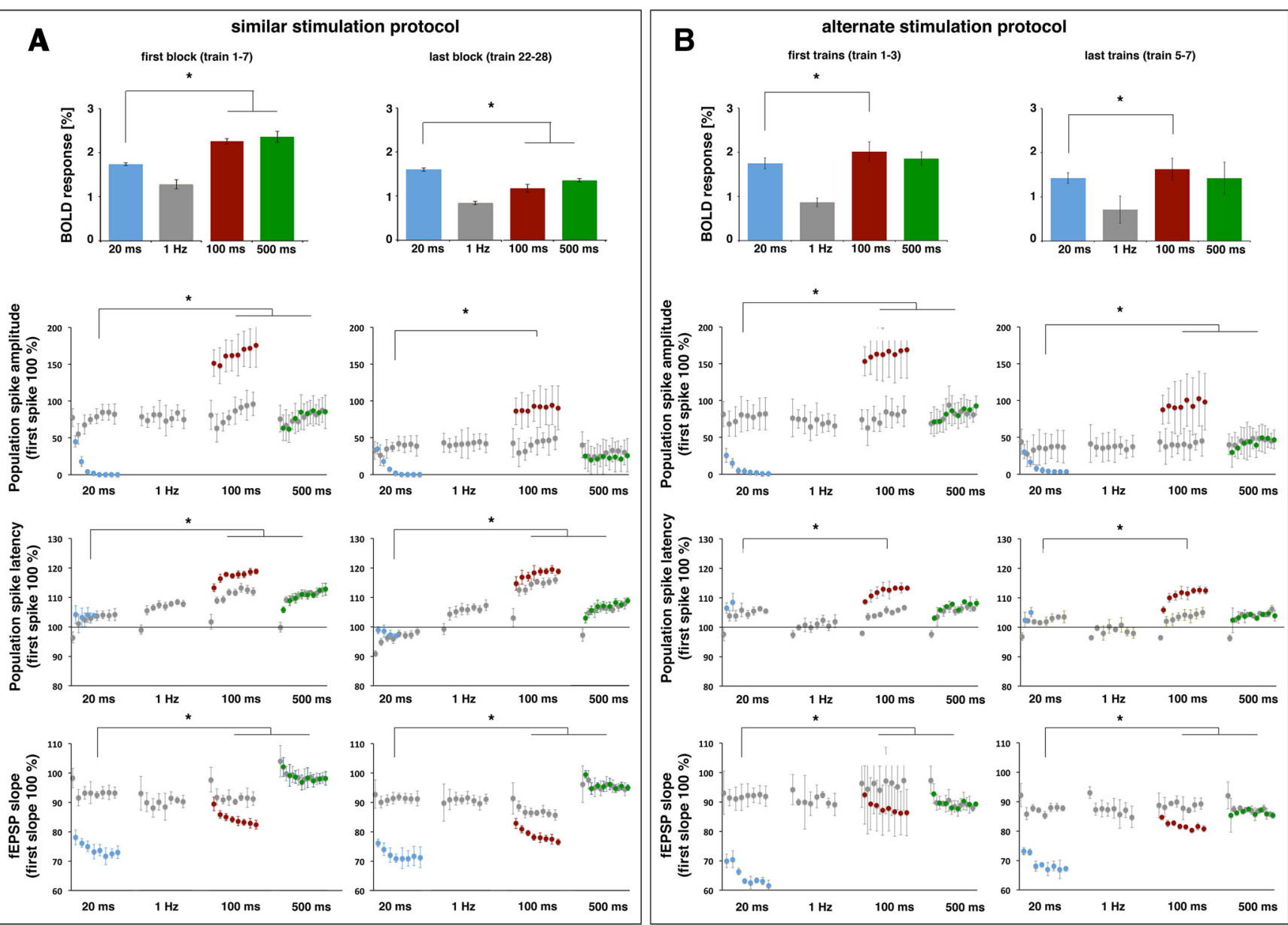

Figure 4. Comparison of generated BOLD responses and the concurrent population spike amplitudes and latencies during different stimulation conditions. $A$, Stimulation of the perforant pathway with the uniform stimulation protocol as depicted in Figure 1A. During the first stimulation block (i.e., trains 1-7), paired-pulse stimulations with an IPI of $20 \mathrm{~ms}$ generated significantly smaller BOLD responses than paired pulses with an IPI of 100 or $500 \mathrm{~ms}$. This paralleled a significantly reduced averaged spiking activity of the granular cells. In addition, the average population spike latency during paired-pulse stimulations with an IPI of $20 \mathrm{~ms}$ was significantly smaller than during the other three conditions. In contrast, during the last stimulation block (train 22-28), paired-pulse stimulations with an IPI of $20 \mathrm{~ms}$ generated significantly stronger BOLD responses than paired pulses with an IPI of 100 or $500 \mathrm{~ms}$. At this time point, the average population spike amplitude during paired pulses with an IPI of $20 \mathrm{~ms}$ was still significantly smaller then during paired-pulse stimulations with an IPI of $100 \mathrm{~ms}$, but similar to the stimulation with continuous $2 \mathrm{~Hz}$ pulses. In contrast to the initial stimulation trains, the average population spike latencies during paired-pulse stimulations with an IPI of $20 \mathrm{~ms}$ were smaller than the initial value (i.e., 100\%). Note that, under this stimulation condition, the average population spike amplitude of the first pulses (gray dots) was similar for all conditions, but the corresponding population spike latencies differed. $\boldsymbol{B}$, Stimulation of the perforant pathway with an alternate stimulation protocol, as depicted in Figure $1 B$. During this condition, paired pulses with an IPI of $20 \mathrm{~ms}$ and continuous $2 \mathrm{~Hz}$ pulses generated similar BOLD responses that were significantly smaller than BOLD responses generated by stimulations with paired pulses with an IPI of $100 \mathrm{~ms}$. This ratio did not change during late stimulation trains. During the alternate stimulation protocol, the average population spike amplitude and latencies of the first pulses (gray dots) remained similar for all conditions; only the responses to the corresponding second pulses (color) were different.

responses to the corresponding second pulses were strongly facilitated; therefore, on average, the population spike amplitude remained at the same level compared with the first block. In contrast to the first set of experiments, the latencies of the first response of the paired-pulse stimulation with an IPI of $100 \mathrm{~ms}$ was only slightly increased $(104.1 \pm 1.3 \%)$; only the latencies of the second responses were clearly increased $(113.6 \pm 1.0 \%)$. As a result, the average population spike latency was reduced compared with the first block (Fig. 5B). The subsequent stimulation with the continuous $2 \mathrm{~Hz}$ protocol resulted in significantly smaller BOLD responses. This was accompanied by a further significantly reduced spiking of the granular cells and significantly increased population spike latency (Table 2). The subsequent paired-pulse stimulation resulted in a similar BOLD response as the previous block, which paralleled significantly increased average population spike amplitudes. Correlating the electrophysiological measured responses with the resultant BOLD responses revealed a close correlation between the popu- lation spike amplitude and the maximal BOLD response $\left(R^{2}=\right.$ $0.708, t_{(26)}=7.939, p<0.0001$; Fig. $\left.5 C\right)$.

When paired-pulse stimulations with an IPI of $30 \mathrm{~ms}$ followed the continuous $2 \mathrm{~Hz}$ protocol, significantly stronger BOLD responses were generated. In addition to the magnitude of the BOLD response, the time to reach this maximum was significantly shorter (Fig. 5A, Table 2). Therefore, the hemodynamic response function (HRF) was shifted to the left. The change in the HRF paralleled a significant reduction of the average population spike amplitude and latency. The subsequently applied $2 \mathrm{~Hz}$ stimulation protocol triggered significantly smaller BOLD responses and a further reduced granular cell-spiking activity than during the previous block. The latencies of the population spikes, however, again increased to $106.9 \pm 0.6 \%$. The following block of paired-pulse stimulation trains then caused significantly stronger BOLD responses than during the last preceding $2 \mathrm{~Hz}$ stimulation block. This paralleled an increased granular cell spiking and reduced population spike latencies (Fig. 5B, Table 2). Correlating 

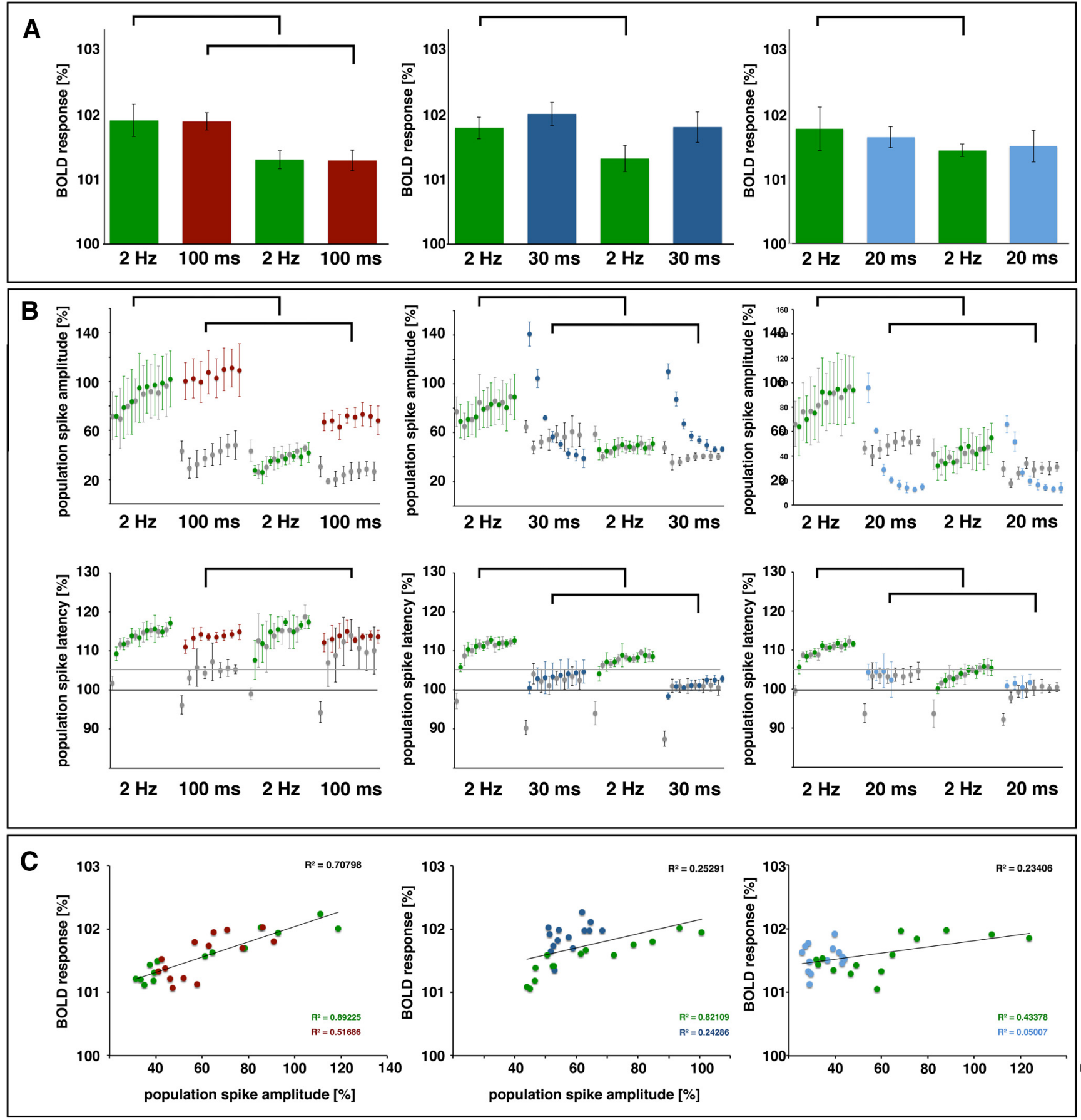

Figure 5. Comparison of generated BOLD responses and the concurrent population spike amplitudes and latencies during two different stimulation conditions. The perforant pathway was again stimulated with four consecutive blocks. The stimulation trains during the first and third blocks contained 16 pulses with a constant IPI of $500 \mathrm{~ms}(2 \mathrm{~Hz})$. The stimulation trains during the second and fourth blocks contained 8 paired pulses with an IPI of $100 \mathrm{~ms}$ (red, left, $n=8$ ), $30 \mathrm{~ms}$ (dark blue, middle, $n=8$ ), and $20 \mathrm{~ms}$ (light blue, right, $n=7$ ). $A$, In all three experiments, the average BOLD responses significantly declined from the first to the third stimulation block. Similarly, the average BOLD response significantly declined from the second to the fourth block when the IPI of the paired pulses was $100 \mathrm{~ms}$. No significant decline of the average BOLD response was found between the second and fourth block when the IPI was 30 or 20 ms. B, Summary of the electrophysiological response. The first pulses are depicted as gray dots and the corresponding second pulse in green ( $500 \mathrm{~ms} I \mathrm{PI})$, red ( $100 \mathrm{~ms} \mid \mathrm{PI})$, dark blue ( $30 \mathrm{~ms} / \mathrm{PI})$, and light blue ( $20 \mathrm{~ms} I \mathrm{IPI})$. In all three experiments, there was a significant decline of the average population spike amplitude between the two identical stimulation blocks. The development of the population spike latency during the $2 \mathrm{~Hz}$ stimulation blocks depended on the applied stimulation protocol. The average latencies remained almost constant when paired-pulse stimulation trains with an IPI of $100 \mathrm{~ms}$ were inserted, decreased by $3.3 \%$ when paired pulses with an IPI of $30 \mathrm{~ms}$ were inserted, and remained on average at a value $>105 \%$ (gray line). The average latency decreased even more (i.e., by $6.2 \%$ ) when paired pulses with an IPI of $20 \mathrm{~ms}$ were inserted and dropped $<105 \%$ on average. C, Correlation of the maximal BOLD response and the average population spike amplitude measured during all 28 stimulation trains. A strong correlation was only present when $2 \mathrm{~Hz}$ stimulation blocks alternated with blocks of paired-pulse stimulations with an IPI of $100 \mathrm{~ms}$. Note that a very strong correlation exists between the maximal BOLD response and the population spike amplitude for all $2 \mathrm{~Hz}$ stimulation trains (indicated by the green value). No clear correlation between these two parameters was found when the $2 \mathrm{~Hz}$ stimulation trains were combined with paired-pulse stimulations with short IPIs. Note that there was still a strong correlation between the maximal BOLD response and the population spike amplitude during all $2 \mathrm{~Hz}$ stimulation trains (indicated by the green value) when paired pulses with an IPI of $30 \mathrm{~ms}$ were inserted (middle row). However, when one block with paired pulses with an IPl of $20 \mathrm{~ms}$ was applied before the second block $2 \mathrm{~Hz}$ stimulation trains (right row), the population spike amplitude only correlated moderately with the maximal BOLD response. This coincides with drop of the population spike latency $<105 \%$ (gray line). 
Table 2. Summary of electrophysiological measured parameters for granular cell spiking activity and the corresponding maximal BOLD responses during electrical stimulations of the perforant pathway

\begin{tabular}{|c|c|c|c|c|c|c|}
\hline & \multicolumn{3}{|l|}{$\begin{array}{l}\text { fMRI } \\
\text { (A) BOLD } \\
\text { (B) } t-\max _{\text {BOLD }}(\%) \\
\end{array}$} & \multicolumn{3}{|c|}{$\begin{array}{l}\text { Electrophysiology } \\
\text { (A) Population spike amplitude (\%) } \\
\text { (B) Population spike latency (\%) }\end{array}$} \\
\hline & $100 \mathrm{~ms}$ & $30 \mathrm{~ms}$ & $20 \mathrm{~ms}$ & $100 \mathrm{~ms}$ & $30 \mathrm{~ms}$ & $20 \mathrm{~ms}$ \\
\hline \multirow[t]{2}{*}{ Block $1(2 \mathrm{~Hz})$} & (A) $1.87 \pm 0.24$ & $1.77 \pm 0.16$ & $1.74 \pm 0.33$ & (A) $87.44 \pm 21.87$ & $79.18 \pm 14.80$ & $83.66 \pm 24.28$ \\
\hline & (B) $10.3 \pm 0.3$ & $10.0 \pm 0.0$ & $10.3 \pm 0.3$ & (B) $113.20 \pm 0.31$ & $110.18 \pm 0.51$ & $109.43 \pm 0.22$ \\
\hline \multirow[t]{2}{*}{ Block (2 pp) } & (A) $1.85 \pm 0.13$ & $\uparrow 1.98 \pm 0.18$ & $1.61 \pm 0.16$ & (A) $72.83 \pm 12.54$ & $\downarrow 62.57 \pm 3.66$ & $\downarrow 41.01 \pm 2.70$ \\
\hline & (B) $10.0 \pm 0.0$ & $\downarrow 8.9 \pm 0.4$ & $\downarrow 9.1 \pm 0.4$ & (B) $\downarrow 108.83 \pm 0.95$ & $\downarrow 102.28 \pm 2.17$ & $\downarrow 103.23 \pm 2.20$ \\
\hline \multirow[t]{2}{*}{ Block (4 pp) } & (A) $1.26 \pm 0.15$ & $\uparrow 1.78 \pm 0.23$ & $1.50 \pm 0.20$ & (A) $\uparrow 47.26 \pm 5.86$ & $\uparrow 52.51 \pm 1.29$ & $\downarrow 28.14 \pm 1.31$ \\
\hline & (B) $10.0 \pm 0.0$ & $9.1 \pm 0.4$ & $8.8 \pm 0.4$ & (B) $110.95 \pm 1.60$ & $\downarrow 100.31 \pm 0.63$ & $\downarrow 100.04 \pm 0.81$ \\
\hline
\end{tabular}

Listed are the average values \pm SD. The maximal BOLD values represent the percentage increase of BOLD signal intensities and the $t$-max ${ }_{\text {BoLD }}$ values represent the time between stimulus onset and maximal BOLD response (in seconds). Al electrophysiological data are relative values and are related to the response to the first pulse $(=100 \%)$. Arrows indicate significant changes $(p<0.05)$ from the previous stimulation block.

the electrophysiological measured responses with the resultant BOLD response revealed no correlation between the population spike amplitude and the resultant BOLD response $\left(R^{2}=0.253\right.$, $\left.t_{(26)}=2.967\right)$. However, if only the responses during the $22 \mathrm{~Hz}$ stimulation blocks were considered, a strong correlation between population spike amplitude and maximal BOLD response was still present $\left(R^{2}=0.821, t_{(12)}=7.421, p<0.001\right.$; Fig. $\left.5 C\right)$.

The change to paired-pulse stimulations with an IPI of $20 \mathrm{~ms}$ resulted in a similar magnitude of the BOLD response as during the previous block of continuous $2 \mathrm{~Hz}$ stimulation trains (Fig. $5 A$ ). Again, under this stimulation condition, the HRF was shifted to the left and, therefore, the time to reach the maximal BOLD signal intensity was significantly shorter (Table 2). Reduced average population spike amplitudes and latencies characterized the corresponding electrophysiological responses during the second block compared with the first block. The subsequent continuous $2 \mathrm{~Hz}$ stimulation protocol triggered significantly smaller BOLD responses. This was not accompanied by significant changes in the average population spike amplitude and latency, which indicates that the population spike latency was significantly smaller during the second continuous $2 \mathrm{~Hz}$ stimulation block than during the initial $2 \mathrm{~Hz}$ stimulation block. The subsequent change to paired-pulse stimulation again resulted in a similar BOLD response, whereas the population spike amplitude and latency again declined significantly. Correlating the electrophysiological measured responses with the resultant BOLD response again revealed no correlation between the population spike amplitude and the resultant BOLD response $\left(R^{2}=0.234\right.$, $\left.t_{(26)}=2.819\right)$. Considering only the responses to the $2 \mathrm{~Hz}$ stimulation trains, only a weak correlation was found between population spike amplitude and maximal BOLD response $\left(R^{2}=\right.$ $0.434, t_{(12)}=3.032, p<0.05$; Fig. $5 C$ ).

In summary, changing the stimulation protocol from 16 pulses with uniform $500 \mathrm{~ms}$ IPIs (or continuous $2 \mathrm{~Hz}$ ) to 8 paired-pulse stimulations resulted in no reduction of the BOLD response as observed during consecutive $2 \mathrm{~Hz}$ stimulation blocks (Fig. 2). Paired-pulse stimulations with an IPI of $100 \mathrm{~ms}$ again resulted in a prolonged latency of the population spikes similar to the $2 \mathrm{~Hz}$ stimulation protocol, an effect that was not seen during paired-pulse stimulation with IPIs of either 20 or $30 \mathrm{~ms}$. According to the development of the population spike latencies, the HRF differed; the time to reach the maximal BOLD signal intensity was longer as soon as the stimulation caused a prolongation of population spike latencies (Table 2). Furthermore, the average BOLD response during the two paired-pulse stimulation blocks with short IPIs (i.e., 20 or $30 \mathrm{~ms}$ ) remained similar, whereas the aver- age BOLD response declined during two paired-pulse stimulation blocks with long IPIs (i.e., 100 ms; Fig. 5A, Table 2).

Electrophysiological and resultant BOLD responses in the dentate gyrus during repetitive stimulations of the perforant pathway with varying paired pulses

In a third set of experiments, the perforant pathway was stimulated during 1 session with 4 different stimulation modalities. In this scenario, 1 train with 8 consecutive paired pulses with an IPI of $20 \mathrm{~ms}$ was followed by 1 train with 8 consecutive single pulses, 1 train with 8 consecutive paired pulses with an IPI of $100 \mathrm{~ms}$, and 1 train with 16 consecutive pulses with a constant IPI of $500 \mathrm{~ms}$ (Fig. 1B). This alternating stimulation protocol was repeated 7 times so 28 trains had been applied by the end.

This stimulation protocol also triggered significant BOLD responses in the right hippocampus (Fig. 6A). The spatial distribution of significantly activated voxels during each individual stimulation condition was similar for all 16 pulse protocols (i.e., 20 and $100 \mathrm{~ms}$ IPIs and continuous $2 \mathrm{~Hz}$ ), whereas for the 8 pulse protocol (i.e., continuous $1 \mathrm{~Hz}$ stimulation), the significantly activated volume was smaller (Fig. 6A).

During this stimulation condition, the population spike amplitudes of the first pulses decreased over time and reached an almost stable level after $\sim 14$ stimulus trains (Fig. $6 B$ ). The responses to the second pulses depended on the IPI; at an IPI of 20 $\mathrm{ms}$, the second pulse always elicited a smaller or no response compared with the corresponding first pulse, whereas at an IPI of $100 \mathrm{~ms}$, the response to the second pulse was always enhanced compared with the corresponding first pulse. The facilitation of the second response was less prominent during later trains than at the beginning. Stimulation with an IPI of $500 \mathrm{~ms}$ resulted in two similar responses. In general, for all stimulation conditions, the development of the individual electrophysiological responses within one train was similar to the responses observed during the first set of experiments.

Similar to the electrophysiological responses, the BOLD responses decreased during consecutive stimulation trains. Whereas the responses to $1 \mathrm{~Hz}$ stimulation trains remained on an almost similar low level, the BOLD responses to consecutive paired pulses and $2 \mathrm{~Hz}$ stimulations were significantly stronger but declined significantly at an almost similar rate. Therefore, a quantitative comparison of the BOLD response magnitude does not critically depend on the chosen number of stimulation trains. During the alternate stimulation protocol, the strongest BOLD response was always induced during paired-pulse stimulation with an IPI of $100 \mathrm{~ms}$. Paired-pulse stimulation with an IPI of 20 


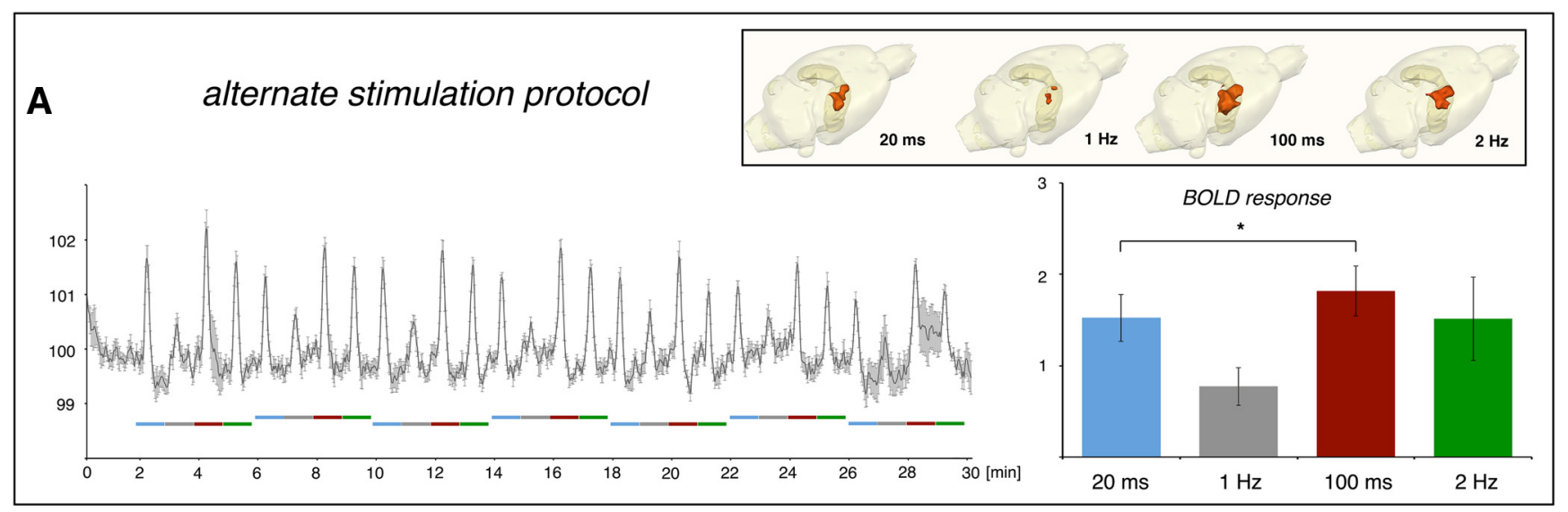

\section{B}
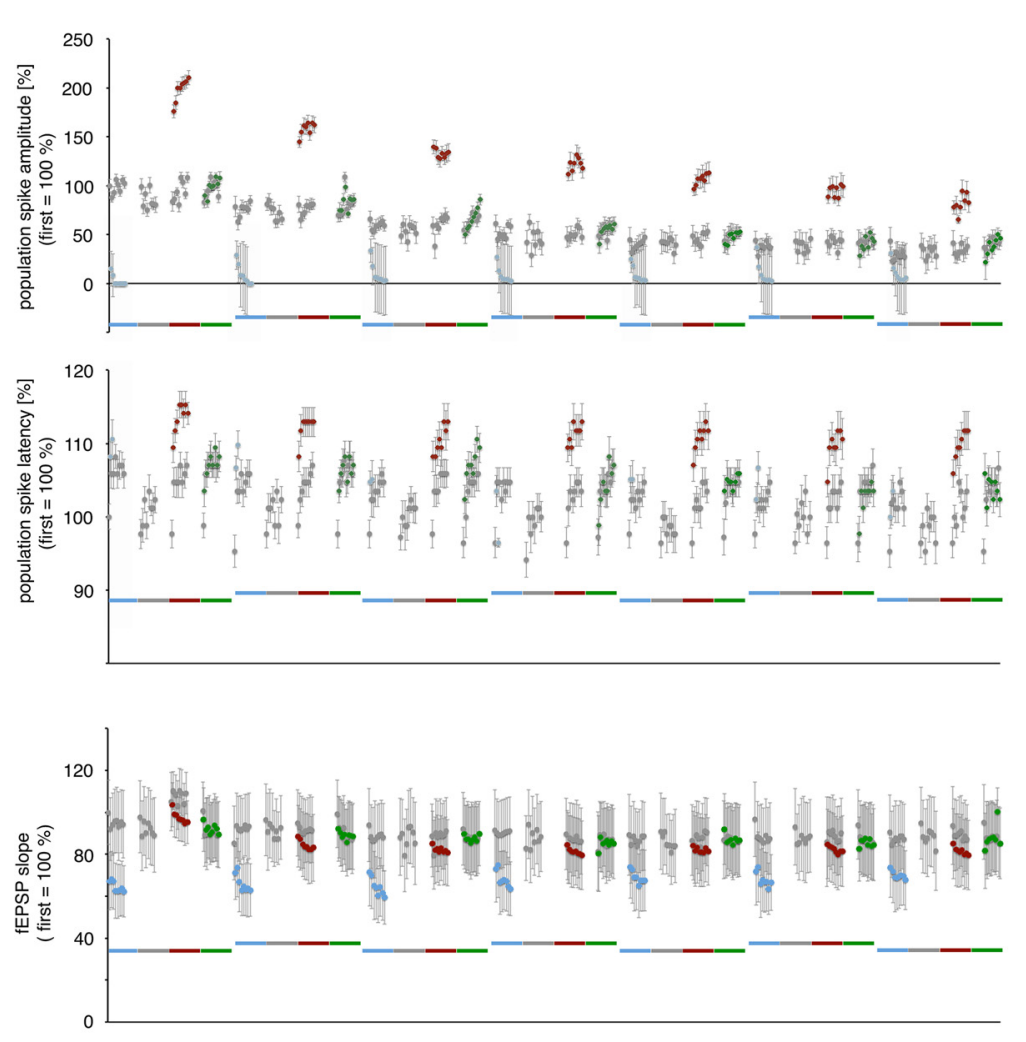
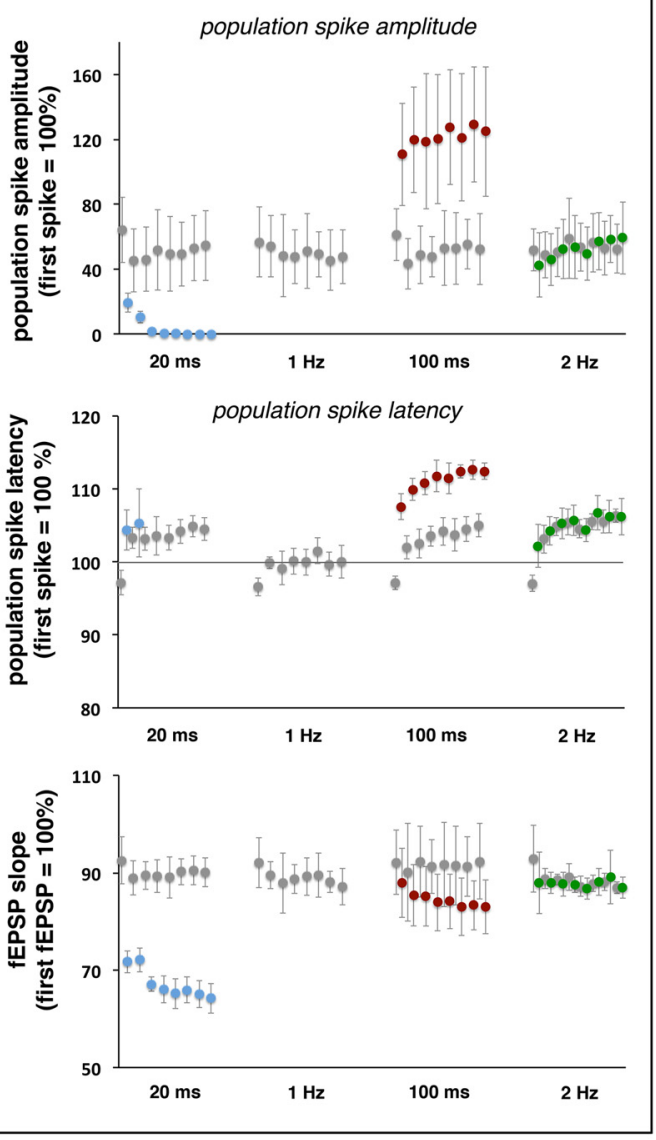
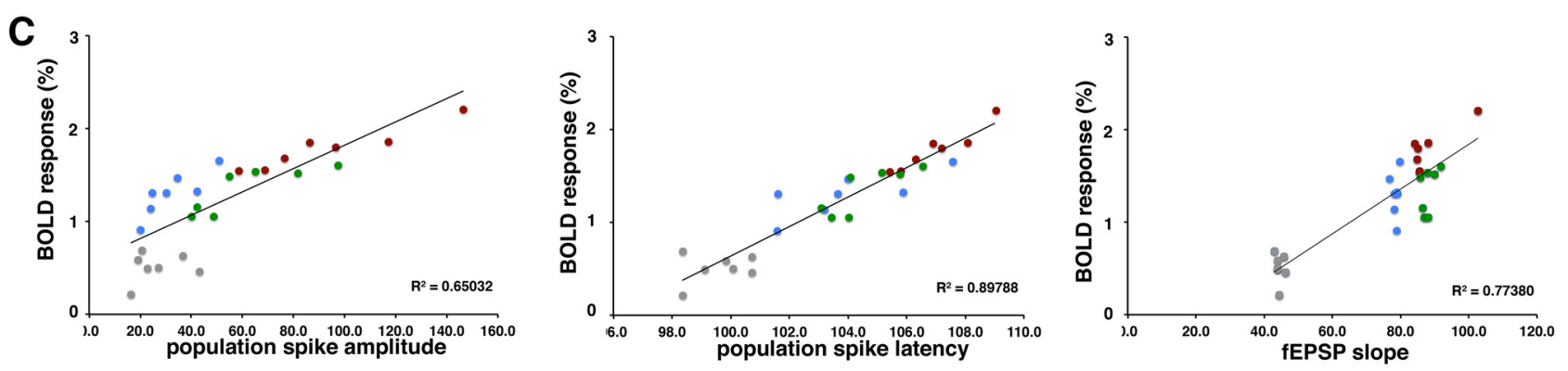

Figure 6. Electrical stimulation of the right perforant pathway with 28 consecutive stimulation trains, as depicted in Figure $1 B$. $A$, The alternate stimulation protocol caused B0LD responses in the right dentate gyrus region that varied in size and magnitude depending on the actual stimulus condition (top, $n=6$ ). The maximal BOLD response was generated during paired-pulse stimulation with $100 \mathrm{~ms}$ IPI. Stimulation with continuous $2 \mathrm{~Hz}$ pulses or paired pulses with an IPI of 20 ms generated similar BOLD responses that were significantly stronger than during continuous stimulations with $1 \mathrm{~Hz}$ pulses. $\boldsymbol{B}$, During consecutive stimulations, the average population spike amplitude of the first pulses (gray dots) per second continuously decreased until train (Figure legend continues.) 
Table 3. Electrophysiologically measured parameters of granular cell activity and the corresponding maximal BOLD responses during repetitive electrical stimulations of the perforant pathway

\begin{tabular}{|c|c|c|c|c|c|c|c|c|}
\hline & \multirow{3}{*}{\multicolumn{2}{|c|}{$\begin{array}{l}\text { fMRI } \\
\text { BOLD signal }\end{array}$}} & \multicolumn{6}{|c|}{ Electrophysiology } \\
\hline & & & \multicolumn{4}{|c|}{ Population spike } & & \\
\hline & & & \multicolumn{2}{|l|}{ Amplitude } & \multicolumn{2}{|l|}{ Latency } & \multicolumn{2}{|l|}{ fEPSP slope } \\
\hline & $\mathrm{BOLD}_{\max }$ & $t-\max _{\mathrm{BOLD}}$ & Pulse 1 & Pulse 2 & Pulse 1 & Pulse 2 & Pulse 1 & Pulse 2 \\
\hline $20 \mathrm{~ms}$ & $1.52 \pm 0.26$ & $9.14 \pm 0.40$ & $51.6 \pm 20.8$ & $3.9 \pm 1.6$ & $103.0 \pm 1.4$ & $104.6 \pm 3.1$ & $90.0 \pm 2.8$ & $67.2 \pm 2.2$ \\
\hline $1 \mathrm{~Hz}$ & $0.77 \pm 0.21$ & $9.43 \pm 0.37$ & $50.0 \pm 17.7$ & & $99.6 \pm 1.0$ & & $89.0 \pm 2.3$ & \\
\hline $100 \mathrm{~ms}$ & $1.82 \pm 0.27$ & $9.43 \pm 0.37$ & $51.8 \pm 17.3$ & $121.4 \pm 36.4$ & $102.8 \pm 1.3$ & $111.1 \pm 1.4$ & $91.6 \pm 7.2$ & $84.5 \pm 5.9$ \\
\hline $2 \mathrm{~Hz}$ & $1.51 \pm 0.46$ & $9.71 \pm 0.29$ & $53.1 \pm 15.8$ & $52.3 \pm 17.5$ & $104.1 \pm 1.1$ & $105.1 \pm 1.7$ & $88.7 \pm 2.0$ & $87.7 \pm 2.3$ \\
\hline
\end{tabular}

and maximal BOLD response (in seconds). All electrophysiological data are relative values and are related to the response to the first pulse (100\%).

ms and the $2 \mathrm{~Hz}$ stimulation protocol resulted in a similar BOLD response (Fig. 6B, Table 3 ).

Relating the spiking activity to the resultant BOLD response revealed a correlation between the population spike amplitude and maximal BOLD response $\left(R^{2}=0.650, t_{(26)}=6.954, p<\right.$ 0.0001; Fig. $6 C$ ). During paired-pulse stimulation with an IPI of $20 \mathrm{~ms}$, we observed a clear dissociation between granular cellspiking activity and the corresponding BOLD response. Due to the strong inhibition of the second response, the average spiking was similar to the $1 \mathrm{~Hz}$ protocol, but the resultant BOLD response was similar to the $2 \mathrm{~Hz}$ protocol (Fig. 6C). In contrast to the population spike amplitude, the population spike latency correlated very closely to the maximal BOLD response $\left(R^{2}=0.898\right.$, $t_{(26)}=15.116, p<0.0001$; Fig. $\left.6 C\right)$. The slope of the fEPSP apparently correlated with the magnitude of the corresponding BOLD response $\left(R^{2}=0.774, t_{(26)}=9.430, p<0.0001\right.$; Fig. $\left.6 C\right)$. Plotting the slope of the fEPSP against the resulting BOLD response revealed a clear separation between the individual stimulation protocols; that is, the same BOLD response but different fEPSP slopes between $2 \mathrm{~Hz}$ stimulation and paired-pulse stimulation with an IPI of $20 \mathrm{~ms}$ and the same fEPSP slope but different BOLD responses between $2 \mathrm{~Hz}$ stimulation and paired-pulse stimulation with an IPI of $100 \mathrm{~ms}$. Therefore, the correlation is mainly caused by the $1 \mathrm{~Hz}$ values. Therefore, considering only the fEPSP slope and the resulting BOLD response for the 32 pulse stimulation protocols, no significant correlation between these two factors was present $\left(R^{2}=0.290, t_{(19)}=2.785\right)$.

In contrast to the first set of experiments, the responses to the first pulses were similar: the population spike amplitude, latency, and slope of the fEPSP were similar in all three 16-pulse stimulation protocols (Table 3 ); only the responses to the corresponding second pulses varied between the three conditions. Therefore, observed differences in the magnitude of the BOLD response can be related to the properties of the population spikes triggered by the second pulses.

In summary, during the alternate stimulation protocol, paired pulses with an IPI of $100 \mathrm{~ms}$ triggered the strongest BOLD responses, whereas paired pulses with an IPI of $20 \mathrm{~ms}$ generated significantly smaller BOLD responses that were similar to the BOLD response induced during $2 \mathrm{~Hz}$ stimulation. The magni-

$\leftarrow$

(Figure legend continued.) 14, whereas the corresponding average population spike latencies and slopes of the fEPSPs remained almost constant. The average response of the first pulse was similar for all four conditions. Depending on the stimulation protocol, the responses to the corresponding second pulses (blue dots: $20 \mathrm{~ms}$ IPI, red dots: $100 \mathrm{~ms} \mathrm{IPI,} \mathrm{green} \mathrm{dots:} 500 \mathrm{~ms}$ IPI) differed in population spike amplitude, latency, and slope of the fEPSP. C, Correlation between maximal BOLD response and various parameters of granular cell activity. tude of the BOLD response correlated best with the concurrent measured average population spike latency.

\section{Discussion}

As expected, an identical amount of input activity to the dentate gyrus causes different BOLD responses because the processing of the incoming signals varies. The most surprising result of the present study was that, depending on the stimulation protocol, the spiking of granular cells on the one side strongly correlated with the resultant BOLD response and on the other side was apparently completely unrelated to the BOLD response. In general, during paired-pulse stimulations that induced a similar or even enhanced granular cell response to the second pulse, the magnitude of a BOLD response strongly correlated with the overall spiking activity of principal neurons. However, as soon as inhibition became effective, the magnitude of the BOLD response clearly dissociated from the spiking activity. Obviously, only during conditions with no effective inhibition of the second pulse are synaptic and spiking activity closely related. Accordingly, the magnitude of the BOLD response could still primarily depend on the amount of postsynaptic activity. However, the slope of the fEPSP, as a measure of postsynaptic activity of the principal cells, only correlated with the magnitude of the BOLD response when the spiking activity also correlated. Even under conditions in which the slopes of the fEPSPs strongly correlated with the spiking activity (i.e., during paired-pulse stimulations with an IPI of $30 \mathrm{~ms}$ ), there was no clear correlation between postsynaptic neuronal activity and the corresponding BOLD response. This indicates either that interneurons, the activity of which is not reflected by the measured field potentials, control the generation of a BOLD response or that there must be another factor in addition to the summed postsynaptic and spiking activity that controls the magnitude of the BOLD response. The only electrophysiologically detectable difference between a correlated and uncorrelated granular cell-spiking BOLD response relationship was the direction in which the average population spike latency developed during consecutive stimulations. When the average population spike latency to the first pulse shortened, the spiking activity was seemingly unrelated to the BOLD response, whereas a strong correlation between the two parameters was always found when the average population spike latency significantly increased by at least $2.2 \mathrm{~ms}$ (i.e., $>105 \%$ ). Furthermore, only during conditions with no obvious relations between spiking activity and BOLD response did the BOLD responses to consecutive stimulations remain almost consistent. Therefore, under this condition, the BOLD response relates to mechanisms upstream of the granular cells that are consistently activated by the applied uniform pulses, such as an activation of the surrounding astro- 

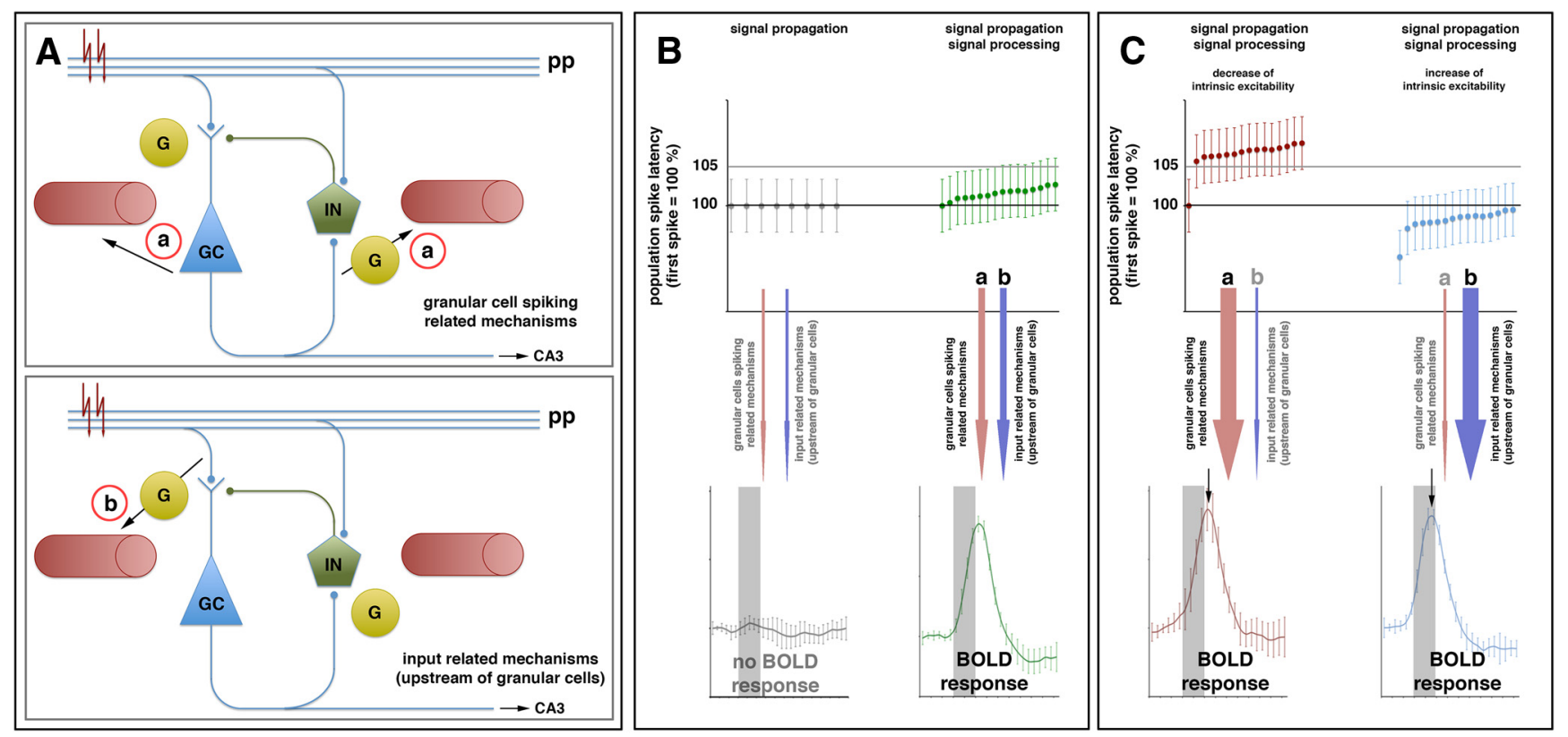

Figure 7. The BOLD response in the dentate gyrus is controlled by at least two different mechanisms. $A$, Electrical stimulation of perforant pathway fibers with paired pulses results in the activation of granular cells (GSS) in the dentate gyrus, which in turn propagate the incoming signals to the CA3 region of the hippocampus proper. GC spiking activates inhibitory interneurons (INs) in the dentate gyrus, which suppresses the GC activity. Some of the INs are also activated directly by perforant pathway fibers. $\boldsymbol{a}$, The spiking of the granular cells, either directly or via glial cells (G), mediates a hemodynamic response that can be detected by fMRI as a BOLD response. $\boldsymbol{b}$, In addition, glutamate released from synaptic terminals of the perforant pathway fibers affects, probably via glial cells, the local hemodynamic parameters. $\boldsymbol{B}$, Very low-frequency stimulation of the perforant pathway (i.e., $<1 \mathrm{~Hz}$ ) elicits substantial spiking of granular cells. Under this condition, repetitive applied pulses cause identical responses (indicated by the constant population spike latencies); therefore, no substantial signal processing occurs. Under this condition, the two neurovascular coupling mechanisms are not efficient enough to trigger a significant BOLD response. Increasing the input activity not only causes spiking of the granular cells, but also an activity-dependent variation in the individual responses (as indicated by an increase of the population spike latency during consecutive pulses). During this condition, a significant BOLD response is generated that may depend on the two mechanisms; that is, it may be controlled by granular cell spiking and the input-related mechanisms illustrated in $A$. C, Repeating the stimulation protocol can modify the intrinsic excitability of the granular cells. When the intrinsic excitability is reduced, a prolongation of the population spike latency is observed ( $>105 \%$ or $>2.2 \mathrm{~ms}$, gray line). Under this condition, the magnitude of the BOLD response mainly depends on the spiking activity of the granular cells. In contrast, when the intrinsic excitability is increased, the population spike latency decreases. During this condition, the magnitude of the BOLD response does not critically depend on the spiking activity of the granular cell, but is more closely related to the input activity. The hemodynamic function characterizing the BOLD response differs between the two conditions; in particular, the time to reach the maximal BOLD signal intensity (black arrow) is shorter when the intrinsic excitability of the granular cells is increased.

cytes by the presynaptically released glutamate. In astrocytes, glutamate mediates the release of various vasodilatory substances, such as arachidonic acid metabolites (e.g., prostaglandin $\mathrm{E}_{2}$ ), epoxyeicosatrienoic acid, and D-serine (Koehler et al., 2009; Park et al., 2011; Petzold and Murthy, 2011; Stobart et al., 2013).

In the experimental setup used here, fibers of the perforant pathway were electrically stimulated and the response of the granular cells was measured. Because the granular cells in the dentate gyrus are only monosynaptically activated by perforant pathway fibers, variations in the spike latency should reflect changes in the excitability of the granular cells; that is, an increased intrinsic excitability will cause shorter population spike latency and a lower intrinsic excitability will cause longer population spike latency. The excitability of granular cells is controlled either synaptically or by their intrinsic properties. Evidently, the population spike latency did not correlate with the fEPSP slope, so the variation in the latency does not depend on changes in the efficacy of the glutamatergic perforant pathway granular cell synapse, but rather reflects a change in the intrinsic excitability of the granular cells.

Based on our observations, we now assume that the actual intrinsic excitability of the activated neurons is one of the critical factors for forming a BOLD response to an incoming stimulus. When previous incoming activations cause increased intrinsic excitability, the subsequently generated BOLD responses are mainly driven by input-related mechanisms (e.g., via astrocytes); in contrast, when previous incoming activations cause a reduced intrinsic excitability, then the subsequently generated BOLD responses are also controlled by postsynaptic and spiking activity (Fig. 7).

This assumption strongly supports the idea of a feedforward mechanism for the generation of a BOLD response in which functional hyperemia is linked to synaptically mediated signaling rather than to the metabolic need of the involved neurons (Attwell et al., 2010; Lindauer et al., 2010; Urban et al., 2012). Only during conditions with a reduced intrinsic excitability can additional factors become effective that relate more to the spiking activity and thus superpose the mainly presynaptic-induced effects (on astrocytes) that affect the local blood flow. Whether the spiking-dependent mechanisms correspond to metabolically related processes or if other astrocyte-mediated mechanisms are responsible remains to be clarified.

Our assumption about at least two independently controlled neurovascular coupling mechanisms originates from experiments in which very simple — but also very artificial—stimulation protocols were used to activate the dentate gyrus. Even under this well defined condition, it is not known to what extent feedback response from other brain regions affect the obtained vascular response and thus modify the relation between the measured electrophysiological responses and resultant BOLD responses. Under normal physiological conditions, a peripheral sensory stimulus evokes a complex neuronal activation pattern (e.g., in the cortex) that may induce in parallel an increase, a decrease, or no change in the intrinsic excitability in subsets of 
neurons. The result is a complex neurovascular coupling process. Because various factors such as previous activations, the actual arousal, attention, and emotional state affect the momentary intrinsic excitability state, the prevalent neurovascular coupling mechanism induced by a constant sensory stimulus may vary considerably. In addition, because synaptic activity is crucial for the two neurovascular mechanisms, but the spiking activity only for certain conditions, the overall synaptic activity appears to be more closely related to the resultant BOLD response then pure spiking activity in most cases (Logothetis et al., 2001). It would, however, be interesting to reevaluate data showing a close relation of fMRI signals to spiking activity, especially in the auditory system (Mukamel et al., 2005), if the majority of neurons displayed a low intrinsic excitability under this circumstance.

The fact that both (pre)synaptically induced signaling mechanisms and, depending on the intrinsic excitability, spikingrelated mechanisms form the vascular response becomes obvious when the results of uniform and alternate pulse stimulation protocols are compared. Application of repetitive trains with uniform paired pulses with an IPI of $20 \mathrm{~ms}$ resulted in shorter population spike latencies than during repetitive stimulation with uniform paired pulses with an IPI of $500 \mathrm{~ms}$ (Table 1). Therefore, according to our assumption, the formation of the BOLD response is on one side mainly controlled by presynapticinduced signaling processes (20 ms IPI) and on the other side mainly by postsynaptic- and spiking-related mechanisms (500 $\mathrm{ms})$. Accordingly, the resultant BOLD responses during the first stimulation train/block were different. We did not record electrophysiological parameters for presynaptic functions. Therefore, putative stimulus protocol-dependent variations in transmitter release and the subsequently induced astrocyte activation cannot be evaluated. Therefore, a direct comparison of the efficacy of the two neurovascular coupling processes is not yet possible. However, based on the different developments of the BOLD response during repetitive stimulations, it appears that under conditions with a reduced intrinsic excitability (i.e., during the $2 \mathrm{~Hz}$ stimulation protocol), the contribution of the presynaptically controlled feedforward mechanism is small. This would explain why the $20 \mathrm{~ms}$ IPI protocol induced a stronger BOLD response than the $2 \mathrm{~Hz}$ stimulation protocol at the end of the stimulation.

In contrast to the uniform stimulation protocol, the alternate stimulation protocol caused similar population spike latencies for the two stimulation conditions (Table 3). Therefore, the intrinsic excitability was similar and corresponded to the condition observed during the initial stimulations with paired pulses with an IPI of $20 \mathrm{~ms}$ (i.e., during the first stimulation block). Accordingly, the magnitude of the BOLD response should relate more closely to the input activity (i.e., 2 pulses per second) and less to the spiking activity. This was indeed the case; the BOLD response was similar, although the spiking activity was significantly different (Fig. 4B, Table 3). Similarly, the usual strong correlation between spiking activity and resultant BOLD response during repetitive $2 \mathrm{~Hz}$ stimulation trains was diminished when trains with paired pulses with an IPI of $20 \mathrm{~ms}$ were interposed, which reduced the average population spike latency during the subsequent $2 \mathrm{~Hz}$ stimulation trains (Fig. 5). Again, the change in population spike latency determined the degree of correlation between spiking activity and the resultant BOLD response.

Our results imply that the magnitude of a BOLD response to a specific incoming stimulus critically depends on the actual intrinsic excitability of the activated neurons. The intrinsic excitability is not only affected for a short time, for example, by previous stimulations, but also for a long time by learning- and memory-related processes or aging (Oh et al., 2003; Matthews et al., 2009; Oh et al., 2010; Sehgal et al., 2013). Therefore, an identical stimulus will cause variable BOLD responses because, in addition to the specific incoming activity, the actual intrinsic excitability of the participating neurons determines the quality of the vascular response. This also implies that, without knowledge of the actual intrinsic excitability of the involved neurons, an assignment of the measured BOLD response to an underlying form of neuronal activity (i.e., presynaptic, postsynaptic, and spiking activity) is exceedingly difficult if not impossible. Therefore, further work has to be aimed at finding specific hemodynamic parameters that relate to only one of these two mechanisms.

\section{References}

Albertson TE, Joy RM (1987) Increased inhibition in dentate gyrus granule cells following exposure to GABA-uptake blockers. Brain Res 435:283292. CrossRef Medline

Angenstein F, Kammerer E, Niessen HG, Frey JU, Scheich H, Frey S (2007) Frequency-dependent activation pattern in the rat hippocampus, a simultaneous electrophysiological and fMRI study. Neuroimage 38:150-163. CrossRef Medline

Angenstein F, Kammerer E, Scheich H (2009) The BOLD response in the rat hippocampus depends rather on local processing of signals than on the input or output activity. a combined functional MRI and electrophysiological study. J Neurosci 29:2428-2439. CrossRef Medline

Angenstein F, Krautwald K, Scheich H (2010) The current functional state of local neuronal circuits controls the magnitude of a BOLD response to incoming stimuli. Neuroimage 50:1364-1375. CrossRef Medline

Attwell D, Buchan AM, Charpak S, Lauritzen M, Macvicar BA, Newman EA (2010) Glial and neuronal control of brain blood flow. Nature 468:232243. CrossRef Medline

Buhl E, Whittington M (2007) Local circuits. In: The hippocampus book (Per Andersen, Richard Morris, David Amaral, Tim Bliss, O’Keefe J, eds), pp 297-319. Oxford: OUP.

Canals S, Beyerlein M, Murayama Y, Logothetis NK (2008) Electric stimulation fMRI of the perforant pathway to the rat hippocampus. Magn Reson Imaging 26:978-986. CrossRef Medline

Hennig J, Nauerth A, Friedburg H (1986) RARE imaging: a fast imaging method for clinical MR. Magn Reson Med 3:823-833. CrossRef Medline

Koehler RC, Roman RJ, Harder DR (2009) Astrocytes and the regulation of cerebral blood flow. Trends Neurosci 32:160-169. CrossRef Medline

Krautwald K, Angenstein F (2012) Low frequency stimulation of the perforant pathway generates anesthesia-specific variations in neural activity and BOLD responses in the rat dentate gyrus. J Cereb Blood Flow Metab 32:291-305. CrossRef Medline

Lindauer U, Dirnagl U, Fuchtemeier M, Bottiger C, Offenhauser N, Leithner C, Royl G (2010) Pathophysiological interference with neurovascular coupling-when imaging based on hemoglobin might go blind. Front Neuroenergetics 2:pii:25. CrossRef Medline

Logothetis NK, Pauls J, Augath M, Trinath T, Oeltermann A (2001) Neurophysiological investigation of the basis of the fMRI signal. Nature 412: 150-157. CrossRef Medline

Lømo T (2009) Excitability changes within transverse lamellae of dentate granule cells and their longitudinal spread following orthodromic or antidromic activation. Hippocampus 19:633-648. CrossRef Medline

Matthews EA, Linardakis JM, Disterhoft JF (2009) The fast and slow afterhyperpolarizations are differentially modulated in hippocampal neurons by aging and learning. J Neurosci 29:4750-4755. CrossRef Medline

McNaughton BL (1982) Long-term synaptic enhancement and short-term potentiation in rat fascia dentata act through different mechanisms. J Physiol 324:249-262. Medline

Mukamel R, Gelbard H, Arieli A, Hasson U, Fried I, Malach R (2005) Coupling between neuronal firing, field potentials, and FMRI in human auditory cortex. Science 309:951-954. CrossRef Medline

Oh MM, Kuo AG, Wu WW, Sametsky EA, Disterhoft JF (2003) Watermaze learning enhances excitability of CA1 pyramidal neurons. J Neurophysiol 90:2171-2179. CrossRef Medline 
Oh MM, Oliveira FA, Disterhoft JF (2010) Learning and aging related changes in intrinsic neuronal excitability. Frontiers in Aging Neuroscience 2:2. CrossRef Medline

Oliver MW, Miller JJ (1985) Alterations of inhibitory processes in the dentate gyrus following kindling-induced epilepsy. Exp Brain Res 57:443447. Medline

Park J, Takmakov P, Wightman RM (2011) In vivo comparison of norepinephrine and dopamine release in rat brain by simultaneous measurements with fast-scan cyclic voltammetry. J Neurochem 119:932-944. CrossRef Medline

Peet MJ, McLennan H (1986) Pre- and postsynaptic actions of baclofen: blockade of the late synaptically-evoked hyperpolarization of CA1 hippocampal neurones. Exp Brain Res 61:567-574. Medline

Petzold GC, Murthy VN (2011) Role of astrocytes in neurovascular coupling. Neuron 71:782-797. CrossRef Medline

Sehgal M, Song C, Ehlers VL, Moyer JR Jr (2013) Learning to learn-intrinsic plasticity as a metaplasticity mechanism for memory formation. Neurobiology of Learning and Memory 105:186-199. CrossRef Medline

Sloviter RS (1991) Feedforward and feedback inhibition of hippocampal principal cell activity evoked by perforant path stimulation: GABAmediated mechanisms that regulate excitability in vivo. Hippocampus 1:31-40. CrossRef Medline

Stobart JL, Lu L, Anderson HD, Mori H, Anderson CM (2013) Astrocyteinduced cortical vasodilation is mediated by $\mathrm{D}$-serine and endothelial nitric oxide synthase. Proc Natl Acad Sci U S A 110:3149-3154. CrossRef Medline

Tuff LP, Racine RJ, Adamec R (1983) The effects of kindling on GABAmediated inhibition in the dentate gyrus of the rat. I. Paired-pulse depression. Brain Res 277:79-90. CrossRef Medline

Urban A, Rancillac A, Martinez L, Rossier J (2012) Deciphering the neuronal circuitry controlling local blood flow in the cerebral cortex with optogenetics in PV::Cre transgenic mice. Front Pharmacol 3:105. CrossRef Medline 